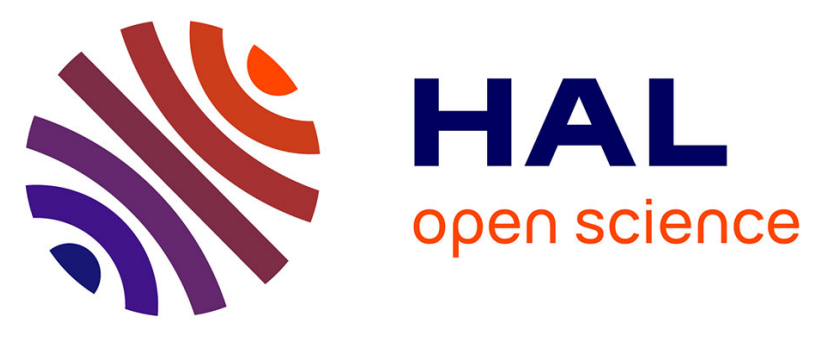

\title{
Mineralocorticoid Receptor Pathway and Its Antagonism in a Model of Diabetic Retinopathy
}

Min Zhao, Emmanuelle Gelize, Rinath Levy, Alexandre Moulin, Frédéric

Azan, Marianne Berdugo, Marie-Christine Naud, Justine Guegan, Kimberley

Delaunay, Eric Pussard, et al.

\section{To cite this version:}

Min Zhao, Emmanuelle Gelize, Rinath Levy, Alexandre Moulin, Frédéric Azan, et al.. Mineralocorticoid Receptor Pathway and Its Antagonism in a Model of Diabetic Retinopathy. Diabetes, 2021, 70 (11), pp.2668 - 2682. 10.2337/db21-0099 . hal-03566169

\section{HAL Id: hal-03566169 \\ https://hal.sorbonne-universite.fr/hal-03566169}

Submitted on 11 Feb 2022

HAL is a multi-disciplinary open access archive for the deposit and dissemination of scientific research documents, whether they are published or not. The documents may come from teaching and research institutions in France or abroad, or from public or private research centers.
L'archive ouverte pluridisciplinaire HAL, est destinée au dépôt et à la diffusion de documents scientifiques de niveau recherche, publiés ou non, émanant des établissements d'enseignement et de recherche français ou étrangers, des laboratoires publics ou privés. 


\title{
Mineralocorticoid Receptor Pathway and Its Antagonism in a Model of Diabetic Retinopathy
} Q:1

\author{
Min Zhao, ${ }^{1}$ Emmanuelle Gelize, ${ }^{1}$ Rinath Levy,, Alexandre Moulin, ${ }^{2}$ Frédéric Azan, ${ }^{3}$ Marianne Berdugo, ${ }^{1}$ \\ Marie-Christine Naud, ${ }^{1}$ Justine Guegan, ${ }^{4}$ Kimberley Delaunay, ${ }^{1}$ Eric Pussard, ${ }^{5}$ Patricia Lassiaz, ${ }^{1}$ \\ Irene Bravo-Osuna, ${ }^{6}$ Rocio Herrero-Vanrell, ${ }^{6}$ and Francine Behar-Cohen, ${ }^{1,3}$
}

Diabetes 2021;70:1-15 | https://doi.org/10.2337/db21-0099

\begin{abstract}
Diabetic retinopathy remains a major cause of vision loss worldwide. Mineralocorticoid receptor (MR) pathway activation contributes to diabetic nephropathy, but its role in retinopathy is unknown. In this study, we show that MR is overexpressed in the retina of type 2 diabetic Goto-Kakizaki (GK) rats and humans and that cortisol is the MR ligand in human eyes. Lipocalin 2 and galectin 3, two biomarkers of diabetes complications regulated by MR, are increased in GK and human retina. The sustained intraocular delivery of spironolactone, a steroidal mineralocorticoid antagonist, decreased the early and late pathogenic features of retinopathy in GK rats, such as retinal inflammation, vascular leakage, and retinal edema, through the upregulation of genes encoding proteins known to intervene in vascular permeability such as Hey1, VIdlr, Pten, Slc7a1, Tjp1, Dlg1, and Sesn2 but did not decrease VEGF. Spironolactone also normalized the distribution of ion and water channels in macroglial cells. These results indicate that MR is activated in GK and human diabetic retina and that local MR antagonism could be a novel therapeutic option for diabetic retinopathy.
\end{abstract}

The worldwide prevalence of diabetes is expected to reach 642 million individuals by 2040 (1), and diabetic retinopathy remains a cause of blindness in the working-age population affecting $50 \%$ of patients with type 1 diabetes and
$30 \%$ of patients with type 2 diabetes after 10 years (2), with no tendency to decrease despite novel therapies (3). Our understanding of diabetic retinopathy has evolved with retinal imaging techniques showing that choroidopathy (4) and reduction of inner retinal layers (ganglion cells [GCs], inner plexiform layer [IPL], and peripapillary nerve fiber layer) may precede the typical microvascular lesions $(5,6)$. Retinal inflammation, oxidative stress (7), and glial cells dysfunction $(8,9)$ are now recognized as major pathogenic factors in ischemic neuronal damages and macular edema, both responsible for vision loss.

Repeated intraocular injections of anti-VEGF drugs that neutralize VEGF family members and intraocular glucocorticoids mostly targeting the inflammatory component reduce macular edema, but they do not benefit all patients and do not target all pathogenic mechanisms of diabetic retinopathy (9), justifying the search for new treatment modalities.

Activation of the mineralocorticoid receptor (MR) pathway plays multiple roles in diabetes complications. It intervenes in early stage of cardiovascular dysfunction during insulin resistance through the local regulation of vascular genes (10). It enhances the inflammasome assembly and subsequent vascular damages in a type 2 diabetic mice model (11). In obese mice, knocking down myeloid MR improves glucose intolerance, insulin resistance, and hepatic
${ }^{1}$ INSERM, From physiopathology of retinal diseases to clinical advances, Centre de Recherche des Cordeliers, Sorbonne Université, Université de Paris, Paris, France

${ }^{2}$ Department of Ophthalmology, University of Lausanne, Jules Gonin Eye Hospital, Fondation Asile des Aveugles, Lausanne, Switzerland

${ }^{3}$ Assistance Publique-Hôpitaux de Paris, Hôpital Cochin Ophthalmopole, Paris, France

${ }^{4}$ Institut du Cerveau, ICM, iCONICS, Hôpital de la Pitié-Salpètrière, Paris, France ${ }^{5}$ INSERM U1185, Hôpital Bicêtre, Université Paris-Saclay, Assistance Publique-Hôpitaux de Paris, Le Kremlin-Bicêtre, France

${ }^{6}$ Department of Pharmaceutics and Food Technology, Instituto Universitario de Farmacia Industrial, Faculty of Pharmacy, Universidad Complutense de Madrid, Madrid, Spain
Corresponding author: Francine Behar-Cohen, francine.behar@gmail.com Received 3 February 2021 and accepted 12 August 2021

This article contains supplementary material online at https://doi.org/10.2337/ figshare.15173394.

(C) 2021 by the American Diabetes Association. Readers may use this article as long as the work is properly cited, the use is educational and not for profit, and the work is not altered. More information is available at https:// www.diabetesjournals.org/content/license. 
steatosis through hepatocyte growth factor (HGF)/Met signaling (12). MR expressed in a number of cells play a role in adiposity, inflammation, and glucose intolerance (13) and its activation in endothelium, smooth muscle cells, inflammatory cells, podocytes, and fibroblasts alters the function and the structure of the diabetic kidney (14). In addition, numerous preclinical and clinical studies have shown that mineralocorticoid receptor antagonist (MRA) is beneficial to reduce the progression of diabetic nephropathy (15) and to reverse diabetic albuminuria (16). Altogether, these results show that MR pathway activation in myeloid cells or in target organs contributes to diabetes-induced tissue damages through oxidative stress, inflammation, fibrosis, and hemodynamic alterations $(14,17)$.

In the retina, overactivation of the MR pathway has been shown to promote inflammation, oxidative stress, and vascular damages (18-21), but its implication in the development of diabetic retinopathy has not yet been explored and the potential benefit of MRA in diabetic retinopathy has not been evaluated.

No animal model recapitulates all the features of human diabetic retinopathy, but Goto-Kakizaki rat (GK), a spontaneous nonobese type 2 diabetic animal, develops progressive retinopathy with local inflammation, retinal circulatory abnormalities (22), and retinal edema $(21,22)$, together with a thickening of the choroid (23), before microangiopathy develops at $\sim 7$ months (24), mimicking the kinetic of events in human diabetic retina (25). Because our aim was to evaluate the effect of MRA on various aspects of retinal edema, we have used this model, as well as an intravitreal microspheres (MSs) formulation of spironolactone, to assess the local role of MRA. Human tissues and samples were also used when possible, to study the role of MR pathway activation in diabetic pathogenesis.

\section{RESEARCH DESIGN AND METHODS}

\section{Animals}

All experiments were performed in accordance with the European Communities Council Directive 86/609/EEC and

Q:2 French national regulations and approved by local ethics committees (no. 2541-2015110210279792 v3, Charles Darwin University). GK rat is a spontaneous nonobese nonhypertensive model of type 2 diabetes, with hyperglycemia as early as 4 weeks of age. Male GK rats of different ages and sex- and age-matched Wistar control rats were included because the inbred GK rat strain is a spontaneous model of type 2 diabetes caused by naturally occurring genetic variants that have been selectively isolated from an outbred colony of Wistar rats (26). GK rats were bred in our animal facility, and Wistar rats were purchased from Charles River Laboratories (Ecully, France). The A1CNow kits (France Neir, Faches-Thumesnil, France) were used to measure the level of $\mathrm{HbA}_{1 \mathrm{c}}$ (\% and $\mathrm{mmol} / \mathrm{mol}$ ). Eightweek-old Wistar rats were included for retinal organotypic culture. Animals were kept in pathogen-free conditions with food, water, and litter and housed in a 12-h light/ 12-h dark cycle. Anesthesia was induced by ketamine $50 \mathrm{mg} / \mathrm{kg}$ i.p. and xylazine $4 \mathrm{mg} / \mathrm{kg}$ i.p. Animals were sacrificed by carbon dioxide inhalation at the end of the experiments.

\section{Intravitreal Injection of Spironolactone-Loaded Poly(lactic-coglycolic) Acid MSs in Rats}

We used spironolactone because it is a very potent MRA (27), whose physicochemical properties allowed the preparation of slow-release polymeric formulations, well tolerated for intraocular use without any risk of systemic side effects $(27,28)$. Spironolactone-loaded poly(lactic-coglycolic) acid (PLGA) (50:50) MSs were elaborated as previously described $(28,29)$. The encapsulation efficiency of spironolactone was $66.28 \%$ (110.45 $\mu \mathrm{g}$ spironolactone/mg MSs), and a constant release rate of $2.87 \mu \mathrm{g}$ spironolactone/mg MSs/day was achieved over 30 days ex vivo. MSs were reconstituted in $0.9 \%$ saline. A single injection of spironolactone-loaded MSs $(5 \mu \mathrm{L}$ containing $11 \mu \mathrm{g}$ spironolactone) was performed in the vitreous of young adult (age 3 months) and old (12-14 months) rats. Control rats were injected with nonloaded PLGA MSs $(5 \mu \mathrm{L})$. Animals were sacrificed after 1 month, and eyes were removed for morphological, immunohistochemical, and quantitative PCR analyses.

To achieve a longer release of spironolactone, we also formulated the MSs from a mixture of Purasorb PDLG 7505 (lactide:glycolide ratio 75:25, mol\%, molecular weight $17 \mathrm{kDa}$; Corbion, Lyon, France) and Purasorb PDL 04 [poly(DL-lactide), molecular weight $45 \mathrm{kDa}$; Corbion]. The drug loading, determined by high-performance liquid chromatography-ultraviolet analysis, was $61.5 \mu \mathrm{g}$ spironolactone/mg MSs (60.4\% encapsulation efficiency). The in vitro release showed close to $100 \%$ sustained release of the active substance over 64 days in $37^{\circ} \mathrm{C}$ PBS (Supplementary Fig. 1). This formulation of spironolactone-loaded MSs was used to assess the gene expression in diabetic retina of old GK rats (age $>1$ year). A single intravitreal injection of $5 \mu \mathrm{L}$ MSs containing $6.2 \mu \mathrm{g}$ spironolactone was performed. After 2 months, retinas were dissected for RNA sequencing. Control rats were injected with nonloaded MSs.

\section{Retinal Pathology}

Enucleated eyes were fixed in $4 \%$ paraformaldehyde and $0.5 \%$ glutaraldehyde for $2 \mathrm{~h}$, dehydrated in a graded alcohol series, and embedded in historesin (Leica, Heidelberg, Germany). Sections of $5 \mu \mathrm{m}$ were obtained with a Leica Jung RM2055 Microtome and stained with 1\% toluidine blue. For quantification, cross sections at the level of optic nerve head were photographed serially, starting from the region adjacent to the optic nerve to the periphery. Measurements of the thickness of the whole retina, IPL, inner nuclear layer (INL), outer nuclear layer (ONL), and the choroid were performed every $300 \mu \mathrm{m}$ from the optic nerve to both sides of peripheral retina with ImageJ ( $n=$ 
4-6 rats per group). GCs were also counted on the entire retinal sections. Measurements of choroidal area and choroidal vascular area were performed on six photographs (125 $\mu \mathrm{m}$ width) taken at every $400-\mu \mathrm{m}$ intervals on each side of optic nerve. Average choroidal and vascular area per photograph was used for comparison.

\section{Immunohistochemistry}

Immunohistochemistry was performed on paraffinembedded retinal sections as previously described (30) ( $n=3-4$ rats per group). The following primary antibodies were used: mouse monoclonal anti-MR 6G1 (1:100; kindly provided by C. Gomez-Sanchez, Division of Endocrinology, University of Mississippi Medical Center, Jackson, MS), rabbit anti-NGAL (1:200, Abcam, Cambridge, U.K.), and mouse anti-galectin 3 (1:200, Santa Cruz Biotechnology, Heidelberg, Germany). Secondary antibodies included

Q:3 biotinylated horse anti-mouse IgG BA2000 (1:250; Vector, Les Ulis, France) and biotinylated goat anti-rabbit IgG BA1000 (1:500; Vector). Procedures for negative controls were performed without primary antibodies.

\section{Immunofluorescence}

Immunofluorescence was performed on retinal cryosections as previously described (30) using rabbit anti-Kir4.1 (1:200; Alomone Labs, Jerusalem, Israel), rabbit antiAQP4 (1:200; Abcam), rabbit anti-IBA1 (1:400; Wako, Neuss, Germany) and goat anti-Brn3a (1:200; Santa Cruz Biotechnology, Heidelberg, Germany) antibodies. Secondary antibodies included Alexa Fluor 488-conjugated goat anti-rabbit IgG (1:200; Thermo Fisher Scientific, Saint Aubin, France), Alexa Fluor 596-coupled goat anti-rabbit IgG (1:200; Thermo Fisher Scientific), and Alexa Fluor 596-coupled donkey anti-goat IgG (1:200; Thermo Fisher Scientific). FITC-coupled sheep anti-rat albumin primary antibody (1:100, Abcam) was also applied to assess vascular albumin leakage on retinal sections of old GK rats $(>1$ year). Procedures for negative controls were performed without primary antibodies.

Fluorescence intensity of AQP4 and Kir4.1 in the inner retina (including nerve fiber layer, GC layer, and IPL), INL, and ONL was measured on retinal sections passing through the optic nerve (12 photographs on each side of optic nerve) with ImageJ software. The corrected total fluorescence was determined by subtracting out background signal: corrected total fluorescence $=$ integrated density (surface of selected area $\times$ mean fluorescence of background). $n=5$ rats per group. Activated round $\mathrm{IBA}^{+}$ microglial/macrophage cells and $\mathrm{Brn3a}^{+}$GCs were also counted on entire retinal sections ( $n=5-6$ rats per group).

For quantification of albumin leakage, the surface of fluorescence (excluding retinal vessels) was quantified on six photographs on the section passing through the optic nerve for each eye ( $n=6$ rats per group) and reported to the total analyzed retinal surface with use of ImageJ software.
Old GK rats (age $>1$ year) were also injected intravenously with FITC-dextran (molecular weight 150,000; Sigma-Aldrich) and then sacrificed when the urine was fluorescent yellow-green, with correct intravenous perfusion indicated. For the quantification of FITC-dextran leakage sites, complete retinal whole mount images were analyzed and the total number of positive sites was quantified ( $n=5$ for nonloaded MSs [NL-MSs]-treated GK group and 8 for spironolactone-loaded MSs [Sp-MSs]-treated group).

\section{Quantitative PCR}

Total RNA from rat neuroretinas was extracted as previously described (30). Transcript levels of genes listed in Supplementary Table 1 were analyzed by quantitative PCR performed in Applied Biosystems QuantStudio 5 Real-Time PCR System (Thermo Fisher Scientific) with SYBR Green detection. Hprt1 and $18 S$ were used as housekeeping genes. $\Delta \mathrm{CT}$ calculation was used for the relative quantification of the results.

\section{RNA Sequencing of GK Rat Retina and Data Analysis}

Two months after intravitreous injection of MSs, the neuroretinas of old GK rats were dissected (three per group). Extracted total RNA samples were sent for sequencing at the iGenSeq transcriptomic platform of the Brain and Spine Institute (ICM) (Paris, France). RNA quality was checked by capillary electrophoresis (Agilent 2100 Bioanalyzer system) and RNA with integrity numbers (RIN) ranging from 7.8 to 8.2 was accepted for library generation. Quality of raw data was evaluated with FastQC. Poor quality sequences were trimmed and adaptors removed with fastp software to retain only good quality paired reads. STAR v2.5.3a (31) was used to align reads on reference genome rn6 using standard options. Between 30 and 38 million reads were mapped. Quantification of gene and isoform abundances was done with rsem 1.2.28 (32), prior to normalization on library size with the edgeR (33) Bioconductor package. Genes with low expression were filtered out. Reproducibility of replicates was controlled with PCA representations. Finally, differential analysis was conducted with the glm framework likelihood ratio test from edgeR. Multiple hypothesis adjusted $P$ values were calculated with the Benjamini-Hochberg procedure to control false discovery rate. The pheatmap Bioconductor package was used to represent as a heat map the differentially expressed genes. The GSEA function from the clusterProfiler package was used on a hallmark gene set to get Q:4 deregulated pathways from analysis, with a representation in a dot plot. A genes/pathways network was created with Cytoscape, with node color representing log fold change.

\section{Excitotoxicity Experiments}

Neuroretinas were dissected from freshly enucleated eyes and then transferred to $0.2-\mathrm{mm}$ polycarbonate membranes (Millipore, Saint-Quentin-en-Yvelines, France) with the GC layer facing up and maintained in DMEM with L-glutamine 
and 5\% serum (Thermo Fisher Scientific). After $24 \mathrm{~h}$, the medium was changed and retinal explants were incubated in medium containing NMDA (100 $\mu \mathrm{g} / \mathrm{mL})$ (M3262; Q:5 SIGMA, France) for $30 \mathrm{~min}$. The medium was then changed again, and explants were either treated with spironolactone $10 \mu \mathrm{mol} / \mathrm{L}$ or vehicle $(0.1 \%$ ethanol) in medium for $48 \mathrm{~h}$. Control explants were incubated with $0.1 \%$ ethanol or spironolactone $10 \mu \mathrm{mol} / \mathrm{L}$ in medium without NMDA exposure. At 24 and $48 \mathrm{~h}$ after NMDA exposure, lactate dehydrogenase (LDH) was measured in the medium $(400 \mu \mathrm{L})$ to determine cytotoxicity using the LDH activity assay (Sigma-Aldrich Chemical Co, Saint-Quentin-en-Yvelines, France). At 48 h, apoptotic cells were detected on retinal explants with the HCS Apoptosis TUNEL Assay (Thermo Fisher Scientific). Nuclei were counter stained with DAPI $(1 / 5,000)$, and the explants were then mounted between two coverslides and pictures were taken with a fluorescence microscope (BX51; Olympus, Rungis, France). TUNEL ${ }^{+}$ cells/retina explant were counted ( $n=4-5$ explants).

\section{Human Biological Samples}

Two eyes from two donors (for science) with type 2 diabetes and moderate retinopathy and four eyes from four donors without diabetes with similar postmortem time were obtained from the Lausanne Eye Bank (CER-VD no. 340/15). Eyes were fixed in $4 \%$ paraformaldehyde overnight. After removal of the cornea and lens, the remaining posterior segment of eyes was dehydrated in a graded alcohol series and embedded in paraffin. Retinal cross sections of $8 \mu \mathrm{m}$ were collected on slides for immunohistochemistry.

The collection and storage of human vitreous were approved by local ethics committee CPP Ile de France 1 (no. 2016-nov-14390). Human vitreous samples $(100 \mu \mathrm{L})$ were collected from patients who had diabetic retinopathy complicated with epiretinal membrane and macular edema or proliferative diabetic retinopathy and underwent vitrectomy. Vitreous samples obtained from patients without diabetes with epiretinal membrane and macular hole were used as control. Only samples collected from noon to 4:00 P.M. were included for steroid profiling to minimize the influence of diurnal variation of steroid hormones. Ten control patients Q:6 without diabetes ( 7 men and 3 women, mean \pm SD age $75.33 \pm 8.15$ years [range 63-88]) and 12 patients with type 2 diabetes (9 men and 3 women, age $76.18 \pm 9.7$ years [60-90]) were included and were not differ with regard to sex ratio or age $(P=0.47)$. Of 12 patients with diabetes, 7 had proliferative diabetic retinopathy and 5 had macular edema with epiretinal membrane and nonproliferative diabetic retinopathy.

\section{Corticosteroid Profiling in Human Vitreous Samples}

The profile of gluco- and mineralocorticoid hormones was established in the vitreous with a highly sensitive and specific liquid chromatographic method coupled with tandem mass spectrometric detection (LC-MS/MS) that allows simultaneous quantification of steroids, targeting the mineralocorticoid (progesterone, 11-deoxycorticosterone, corticosterone, 18-hydroxycorticosterone, and aldosterone) and glucocorticoid (17-hydroxyprogesterone, 11deoxycortisol, cortisol, and cortisone) pathways as previously described (34). Corticosteroid 11- $\beta$-dehydrogenase isozyme $2(11 \beta-H S D 2)$ is an MR protecting enzyme that oxidizes the active cortisol to the inactive metabolite cortisone, thus preventing illicit MR activation. By determining the product-to-substrate ratio (cortisone/cortisol), we compared the $11 \beta-H S D 2$ activity that reflects cortisol accessibility to MR between diabetic and nondiabetic retinas.

\section{Statistics}

Quantitative data were expressed as mean \pm SD. Statistical analysis was made using the GraphPad Prism 8 program (GraphPad Software, San Diego, CA). Nonpaired Student $t$ test or nonparametric Mann-Whitney $U$ test was used to compare two groups. Nonparametric Kruskal-Wallis test followed by a Dun test was used to compare more groups. Grouped data sets were analyzed with multiple $t$ tests, corrected for multiple comparisons with the Holm-Sidak method. $P<0.05$ was considered significant.

\section{Data and Resource Availability}

Data generated during the current study are available from the corresponding author upon reasonable request. RNA-sequencing data are available on Gene Expression Omnibus (GEO): GSE163255 (https://www.ncbi.nlm.nih. gov/geo/query/acc.cgi?acc=GSE163255).

\section{RESULTS}

\section{MR in the Rat and Human Diabetic Retina}

In GK rats, the first sign of retinopathy was a dilation of the choroidal vessels observed at 3 months (Supplementary Fig. 2A-D), associated with thickening of the outer retina $0: 8$ witnessing retinal edema at 6 months (Supplementary Fig. $2 E-G)$. After 12 months, retinal edema extended to all retinal layers with focal accumulations of subretinal fluid (Supplementary Fig. $1 H$ ). With diabetes and aging, cataract becomes occlusive, preventing accurate functional analysis.

$N r 3 c 2$ expression (encoding the MR) was upregulated at 3 and 6 months in the diabetic GK retinas as compared with the Wistar nondiabetic ones. At 12 months, $N r 3 c 2$ expression in Wistar rat retinas was also increased to the same level as in GK rat retinas (Fig. 1A). Immunostaining showed localization of MR in the nuclei of GCs and in cells in the INL, with enhanced labeling in the GK rats at 6 and 12 months without major change in its distribution (Fig. 1B).

In human nondiabetic retinas, MR was localized in the nuclei of GCs, in the INL, and in nuclei and inner segments of cone photoreceptors, which are enriched in the central retina (Fig. 1C). In the diabetic human retina, MR staining was more intense, and more cells were stained in the 


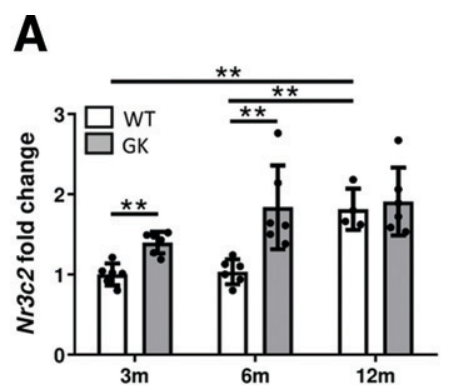

B
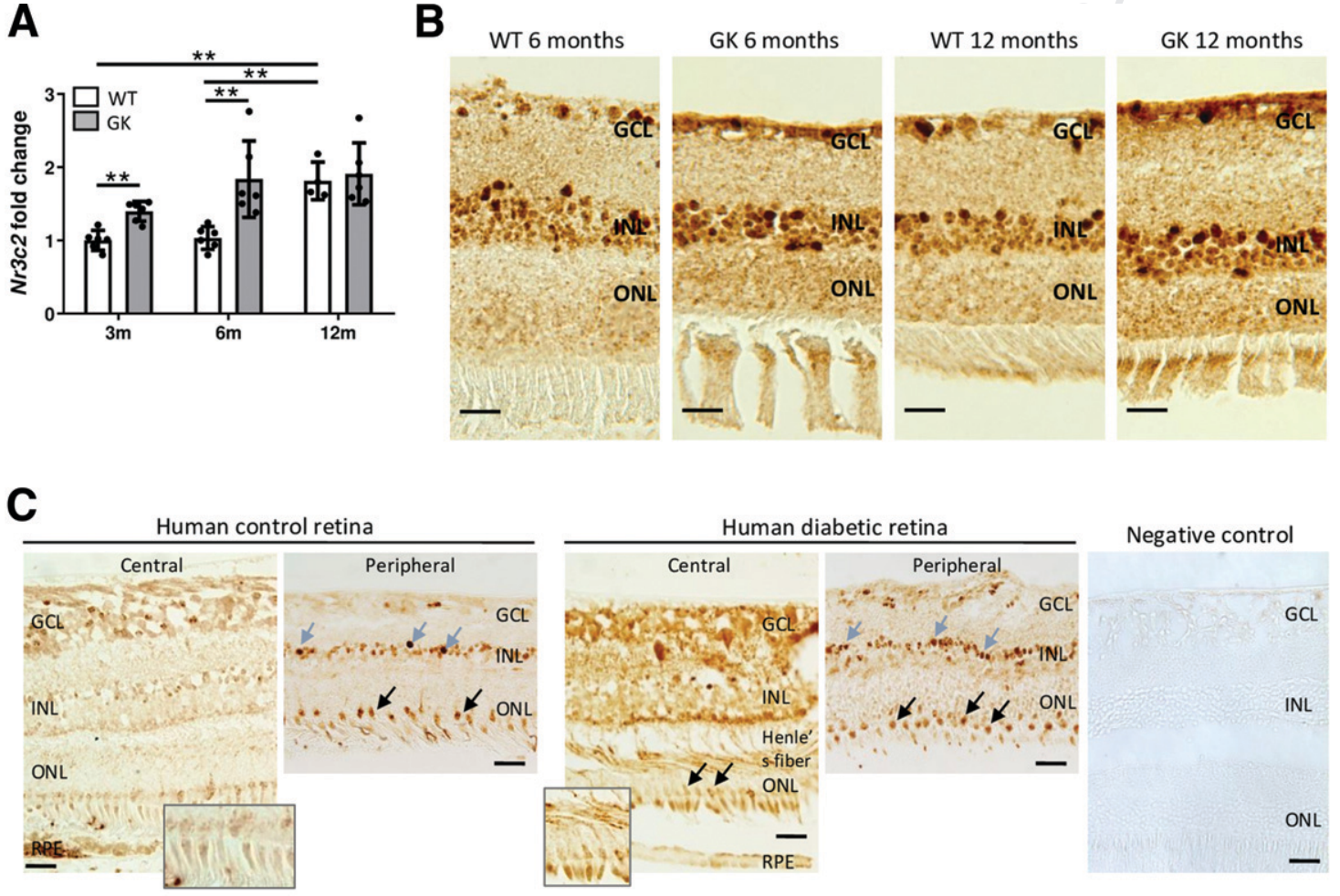

Negative control
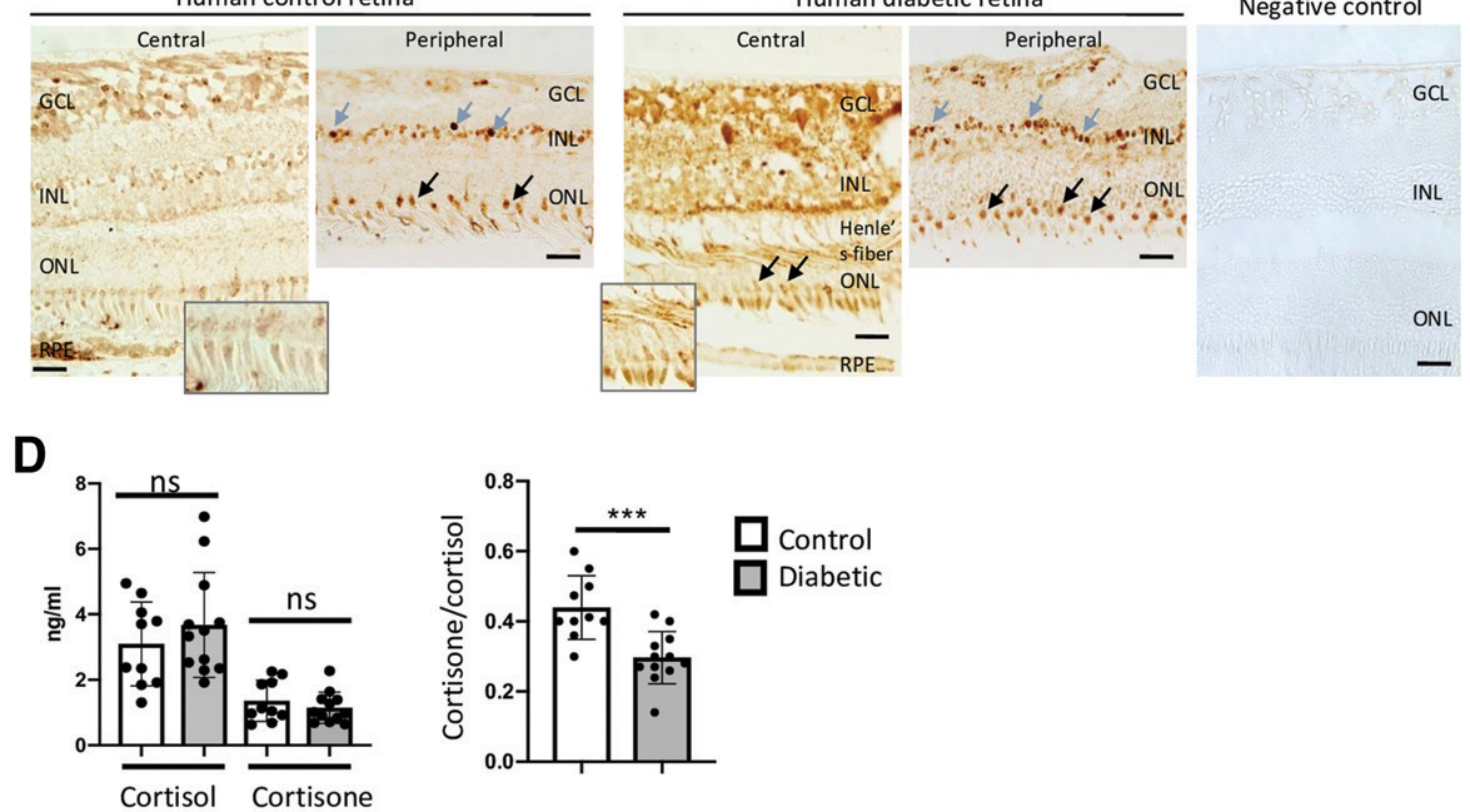

Figure 1-MR expression in diabetic retinas from rat and human and corticosteroid measurement in human vitreous. A: Quantitative PCR shows that $\mathrm{Nr} 3 \mathrm{c} 2$ gene expression is upregulated in the retinas of GK diabetic rats at 3 and 6 months as compared with age-matched nondiabetic Wistar (WT) rats. At 12 months, there is no difference between Wistar and GK rats, as Nr3c2 expression is also upregulated in the retinas of Wistar rats with aging. $n=4-6$ rats per group. Data are expressed as means \pm SD. Hprt1 and $18 S$ are used as housekeeping genes. ${ }^{* *} P<0.01$ (multiple $t$ tests, corrected for multiple comparisons with the Holm-Sidak method). B: Immunohistochemistry shows

Q:25 enhancement of MR protein expression in the retinas of GK rats ages 6 and 12 months as compared with age-matched Wistar rats, particularly in the cells in the INL and GC layer (GCL). Bars: $50 \mu \mathrm{m} . n=3-4$ rats per group. C: In human retinas $(n=4)$, MR is located not only in the nuclei of cells in the INL (gray arrows) and GCL layers but also in the nuclei of cone photoreceptors (black arrows) and their segments

Q:26 (insets). In patients with diabetes $(n=2)$, MR expression is enhanced, particularly in the central retina. Negative control was performed without primary antibody. Bar: $50 \mu \mathrm{m}$. D: Corticosteroid measurement using the human vitreous shows that there is no significant difference (ns) in concentration of cortisol and cortisone in the vitreous of patients with diabetes vs. patients without diabetes. The cortisoneto-cortisol ratio, which represents the activity of the enzyme $11 \beta-H S D 2$, is significantly lower in the vitreous of patients with diabetes than in control subjects without diabetes. $n=10$ in control group and 12 in the group with diabetes. Data are expressed as mean \pm SD. $* * * P<0.001$ (nonpaired Student $t$ test). $\mathrm{m}$, months.

ganglion and inner nuclear cell layers of the peripheral retina as well as in cone photoreceptors in the macula (Fig. 1C).

\section{Cortisol Is the MR Ligand in the Human Diabetic Eyes}

With use of a sensitive LC-MS/MS method, aldosterone was not detected $(<10 \mathrm{pg} / \mathrm{mL})$ in any of the vitreous samples from either diabetic or nondiabetic eyes. But while vitreous cortisol levels did not differ between control and diabetic eyes, the mean \pm SD cortisone-to-cortisol ratio was significantly lower in subjects with diabetes $(0.29 \pm 0.07$ vs. $0.44 \pm 0.09, P=0.008)$, reflecting a lower activity of $11 \beta-H S D 2$ and thus a higher availability of cortisol to MR in diabetic eyes (Fig. 1D). Increased MR and cortisol availability in diabetic eyes suggest hyperactivation of MR by cortisol. 


\section{Local Slow Release of Spironolactone Reverses Early Changes Observed in the GK Rat Retinas}

To compensate for the poor ocular bioavailability of systemic spironolactone and evaluate its direct effect on the retina, we injected in the vitreous of 3-month-old GK rats the PLGA MSs that release spironolactone for at least 1 month (28). No change in $\mathrm{HbA}_{1 c}$ levels was measured (Supplementary Fig. 1C). After a month, dilatation of the choroidal vessels, which was the first sign of retinopathy, was reversed in the spironolactone-loaded MSs (Sp-MSs)treated eyes as compared with the nonloaded MSs (NL-MSs)-treated eyes $(P=0.0152)$ (Fig. $2 A-E)$. The perivascular distribution of the inwardly rectifying potassium channel Kir4.1 and of the water channel aquaporin 4 (AQP4), strongly reduced in the NL-MSs-treated diabetic GK rats, was restored in the retinas of Sp-MSs-treated GK rats (Fig. 2F-M). In addition, the number of activated and displaced $\mathrm{IBA}^{+}{ }^{+}$microglia/macrophage cells was significantly reduced by the Sp-MSs treatment $(P=0.0317)$
(Fig. 3A-E), with a reduction of retinal inflammation. At this early stage of retinopathy, Sp-MSs reduced the expression of placental growth factor (Plgf) and of $\mathrm{Ccl} 2$ and Icam-1 (Fig. 3F), two inflammatory mediators that strongly correlate with diabetic macular edema $(35,36)$ The expression of $I L-18$, witnessing the pathogenic activation of inflammasome (37), was upregulated in GK rat Q:9 retinas but was not decreased significantly with the Sp-MSs treatment (Fig. 3F). At this stage, Vefga expression did not increase in the GK rat retinas and was not downregulated by Sp-MSs (Fig. 3F). Sp-MSs thus reversed some early clinical and molecular signs of diabetic retinopathy in GK rat.

\section{Local Slow Release of Spironolactone Reverses Retinal Edema in 1-Year-Old Diabetic GK Rats}

We then evaluated the effect of spironolactone on retinal edema by injection of the Sp-MSs or NL-MSs into 1-year old GK rat eyes. At this age, edema was

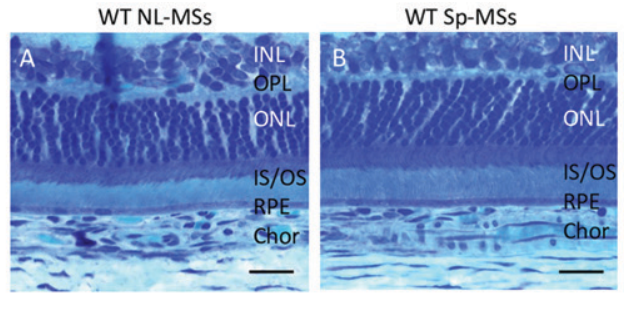

WT
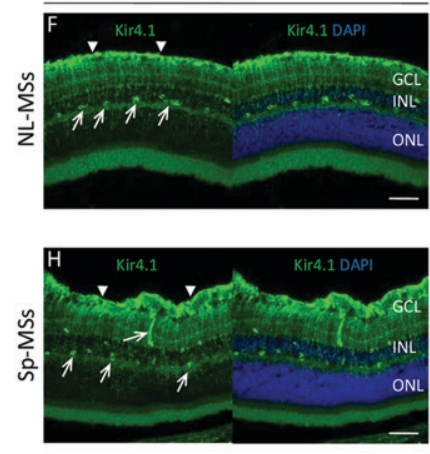

GK NL-MSs

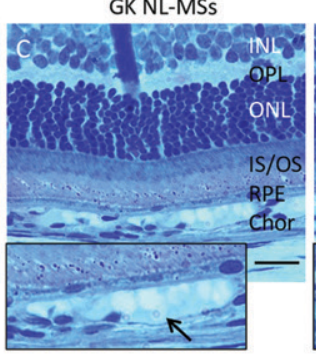

GK
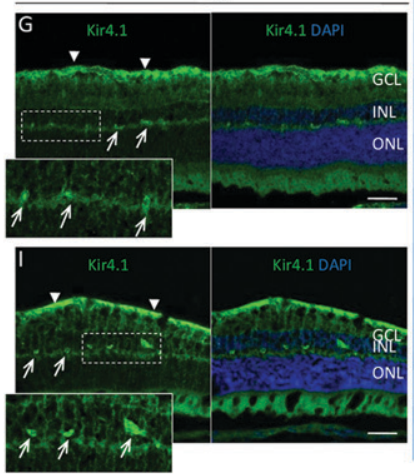

GK Sp-MSs

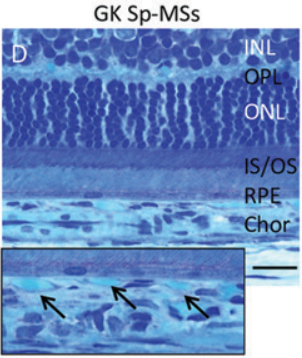

WT
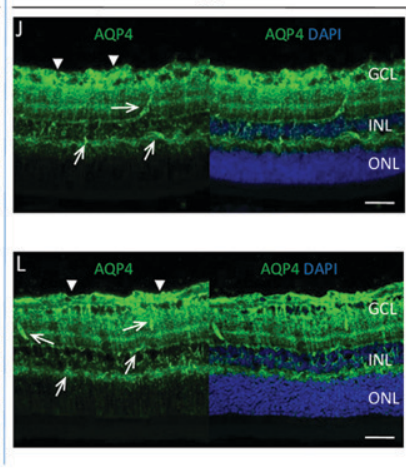

F3 

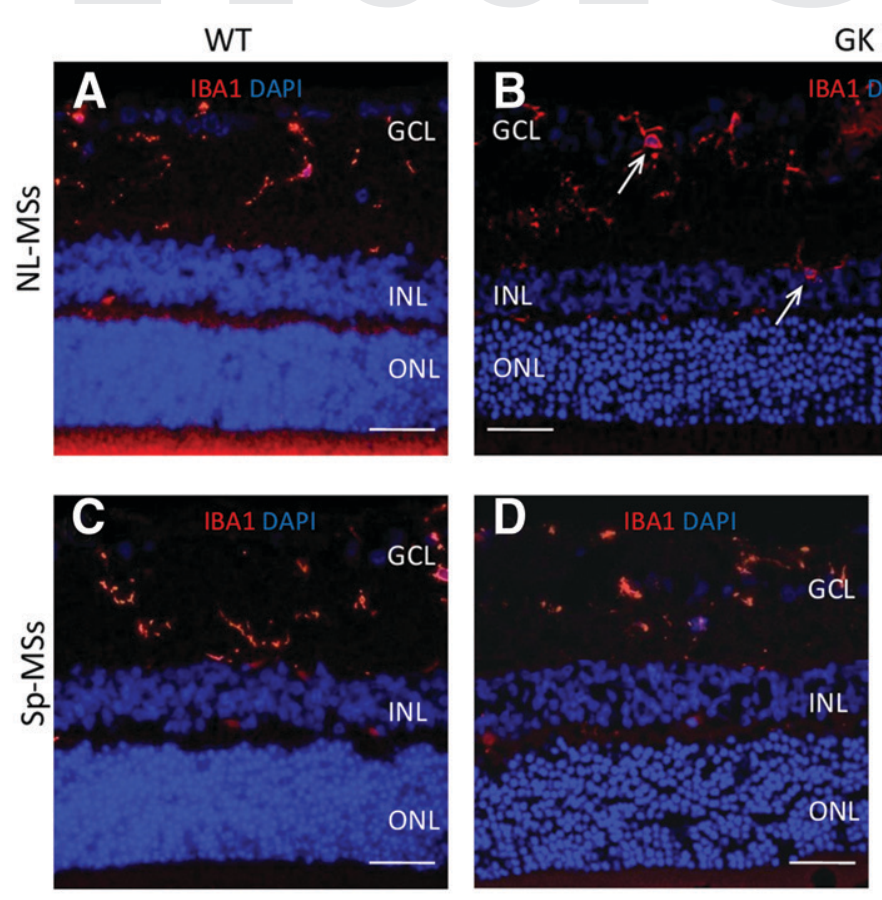

GK
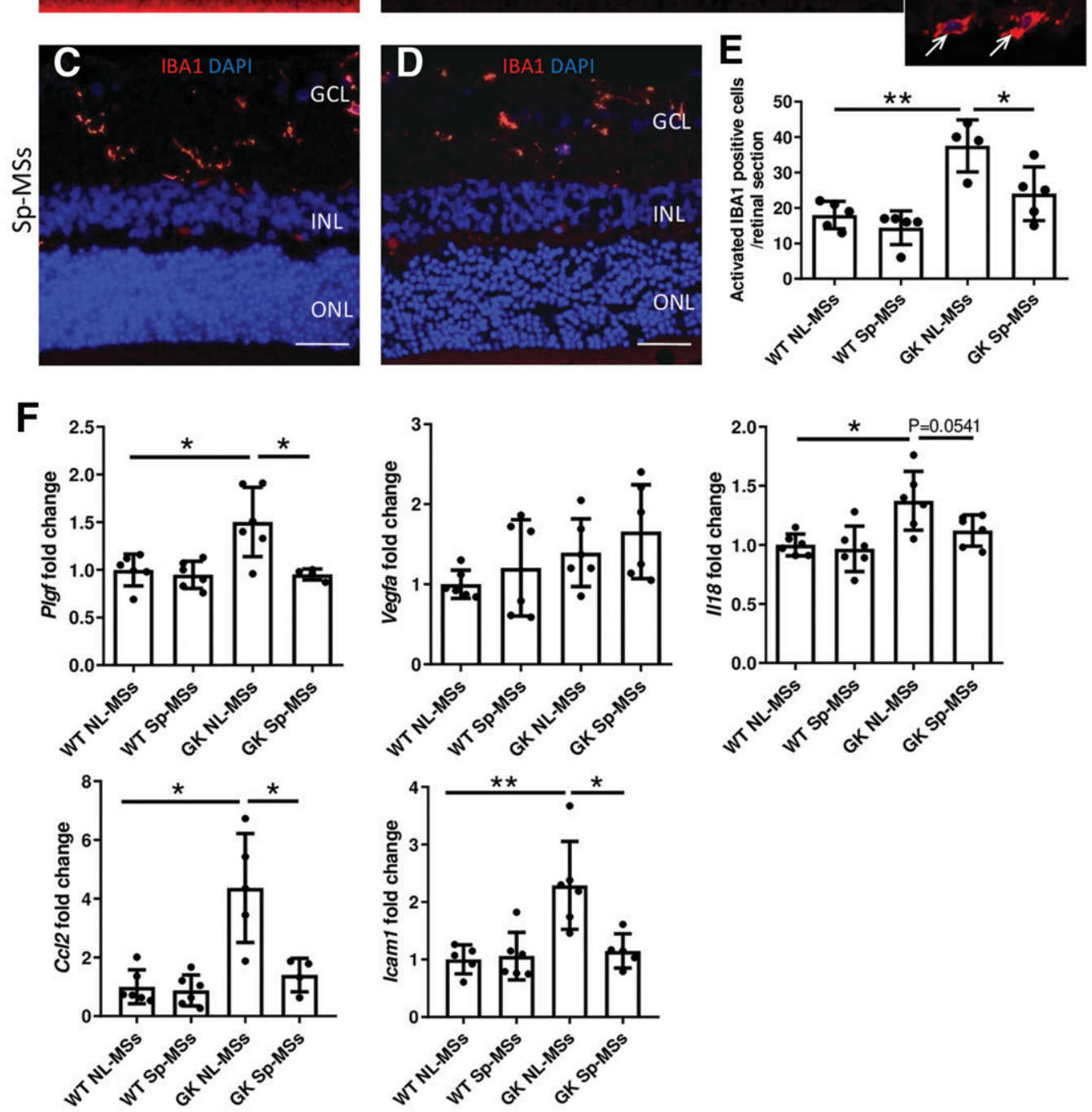

Figure 3-Sp-MSs reduce the early retinal inflammation in the diabetic retinas of GK rats. A-D: IBA1 immunostaining (in red) of microglia/ macrophages in the retinas of 4-month-old Wistar (WT) and GK rats 1 month after intravitreous injection of Sp-MSs and NL-MSs. IBA1 ${ }^{+}$ microglia/macrophages in the retina of WT rats injected with NL- or SP-MSs show ramified processes $(A$ and $C)$. In the retinas of GK rats injected with NL-MSs, round activated IBA $1^{+}$cells (arrows) can be observed in the inner retina and migration of cells can be seen in the ONL and up to subretinal spaces (inset, $B$ ). In the retinas of GK rats treated with Sp-MSs, IBA $1^{+}$cells are in a nonactivated ramified form and locate mostly in the inner retina $(D)$. GCL, GC layer. Bar: $50 \mu \mathrm{m}$. E: Quantification of activated IBA $1^{+}$microglia and macrophages on the retinal sections shows significant increase in number of inflammatory cells in the diabetic retinas of GK rats that is reduced in Sp-MSs-treated GK rats. $n=4-5$ rats per group. Data are expressed as mean \pm SD. $* P<0.05, * * P<0.01$ (Kruskal-Wallis test). F: Quantitative PCR shows upregulation of Plgf and I/18 in the neural retina of diabetic GK rats, while there is no difference in Vegfa expression. SpMSs significantly downregulate plgf. Expression of proinflammatory genes Cc/2 and Icam 1 is also significantly upregulated in the retinas of GK rats and is reduced by the treatment of Sp-MSs. $n=5-7$ rats per group. Data are expressed as mean \pm SD. Hprt1 and $18 S$ are used as housekeeping genes. $* P<0.05, * * P<0.01$ (Kruskal-Wallis test). 
Q:10 more pronounced at the posterior retina resulting from fluid accumulation in the INL, IPL and ONL

(Fig. $4 A-D$ ), mostly on one side on the optic nerve, and choroidal thickness was enlarged on one side around the optic nerve (Fig. 4C). Ocular spironolactone did not influence $\mathrm{HbA}_{1 c}$ levels in old GK rats (Supplementary Fig. 1C), but 1 month after the injection, Sp-MSs significantly reduced retinal edema in the thickened layers of the posterior retina as compared with NL-MSs (Fig. 4A-I) and in the corresponding choroidal thickened region around the optic nerve. The cellular distribution of Kir4.1 and AQP4 was located at the end feet of glial Müller cells, in the inner retina, and faintly distributed along the retinal vessels in the old GK rat (Fig. $5 A, B, E$, and F). Sp-MSs enhanced the Kir4.1 straining around the vessels in the INL and toward the outer limiting membrane (Fig. $5 C$ and $D$ ) and significantly increased AQP4 fluorescence in the INL where deep capillary network was located, and in the ONL (Fig. $5 E-H$ and $J$ ), demonstrating that the treatment favored AQP4 proper relocation. Activated microglia/macrophages, widely distributed in all retinal layers in the 1-year-old GK rats treated with NL-MSs, were significantly decreased by Sp-MSs treatment (Fig. 6A-C). Spironolactone reduced edema through anti-inflammatory effects and normalization

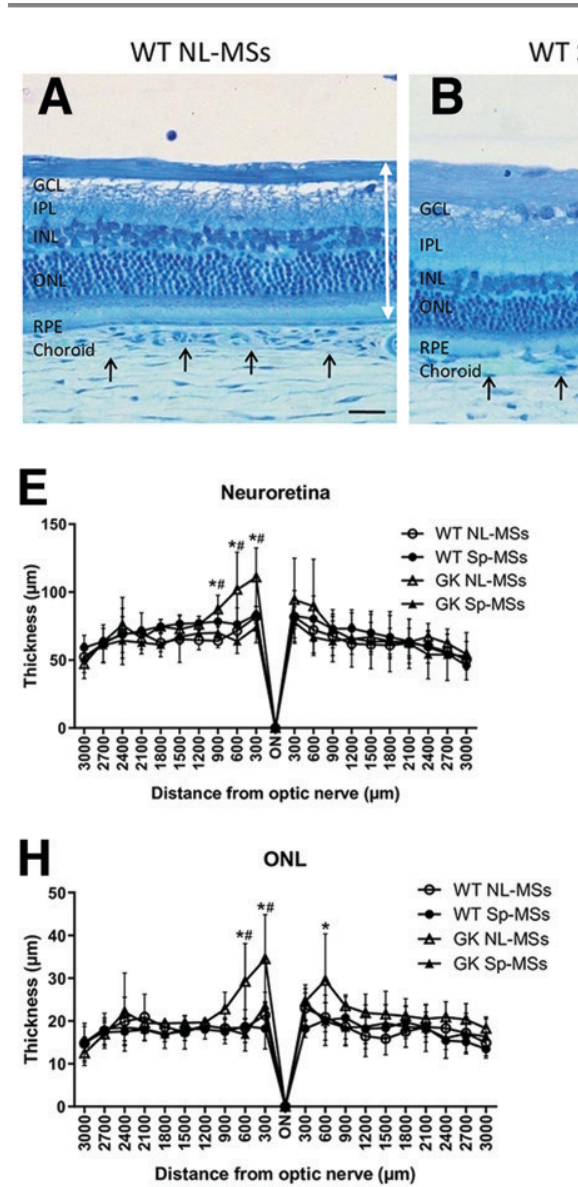

WT Sp-MSs

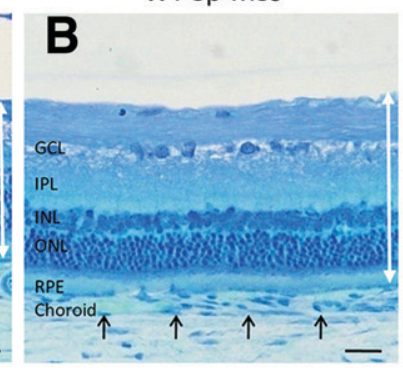

$\mathbf{F}$
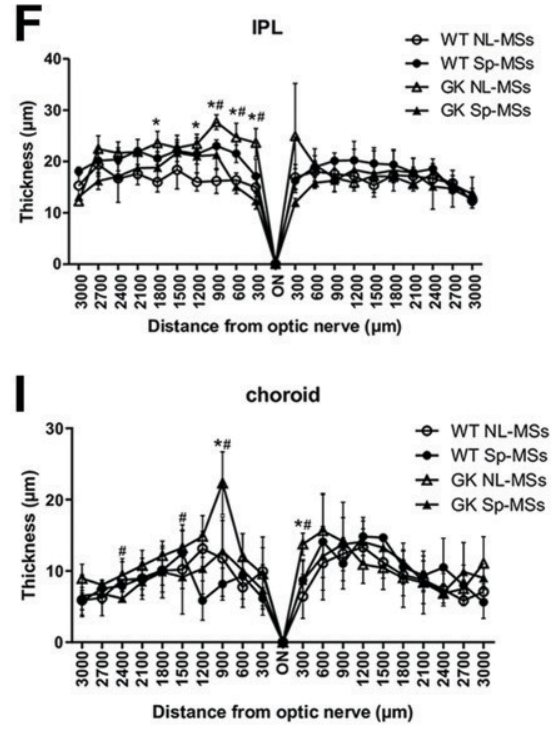

Figure 4-Sp-MSs reduce retinal edema in old GK rats. A-D: Retinal histology of 1-year-old Wistar (WT) and GK rats 1 month after intravitreous injection of NL-MSs or Sp-MSs. Histological sections were stained with $1 \%$ toluidine blue. $A$ and $B$ show the retinal and choroidal structure of old WT rats. The white double-headed arrows indicate the total neuroretinal thickness, and the black arrows indicate the limit of the choroid from the sclera. The diabetic retinas of old GK rats injected with NL-MSs show an increase in retinal thickness at the posterior retina resulting from fluid accumulation in the INL, IPL, and ONL. Red blood cells are accumulated in the dilated choroidal vessels $(C)$. In the retinas of GK rats injected with Sp-MSs, retinal edema and choroidal vascular dilation are reversed $(D)$. GCL, GC layer. Bar: $20 \mu \mathrm{m}$. E-l: Quantification of the total thickness of the neuroretina and the thickness of the IPL, INL, ONL and choroid in WT and GK rats after NLor Sp-MSs intravitreous injection. A significant increase in the total neuroretinal thickness and the thickness of each measured layer is observed at the posterior pole of the diabetic retinas of GK rats treated with NL-MSs, mostly on one side of the optic nerve. Retinal thickness is decreased in the retinas of GK rats treated with Sp-MSs. $n=4$ rats per group. Data are expressed as means \pm SD. $* P<0.05$ between Wistar NL-MSs and GK NL-MSs, \#P $<0.05$ between GK Sp-MSs and GK NL-MSs (multiple $t$ tests, corrected for multiple comparisons with the Holm-Sidak method). ON, optic nerve. 


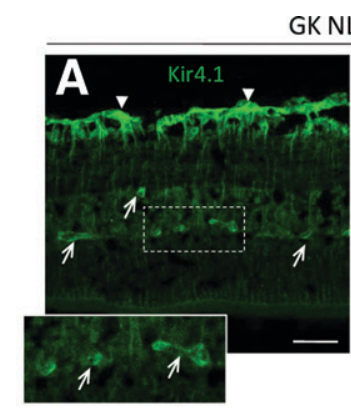

GK NL-MSs

GK Sp-MSs
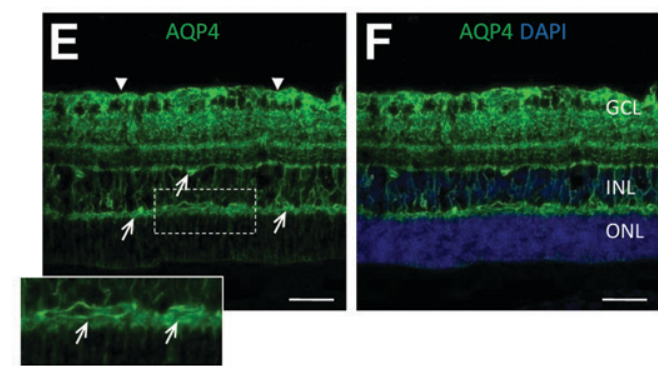
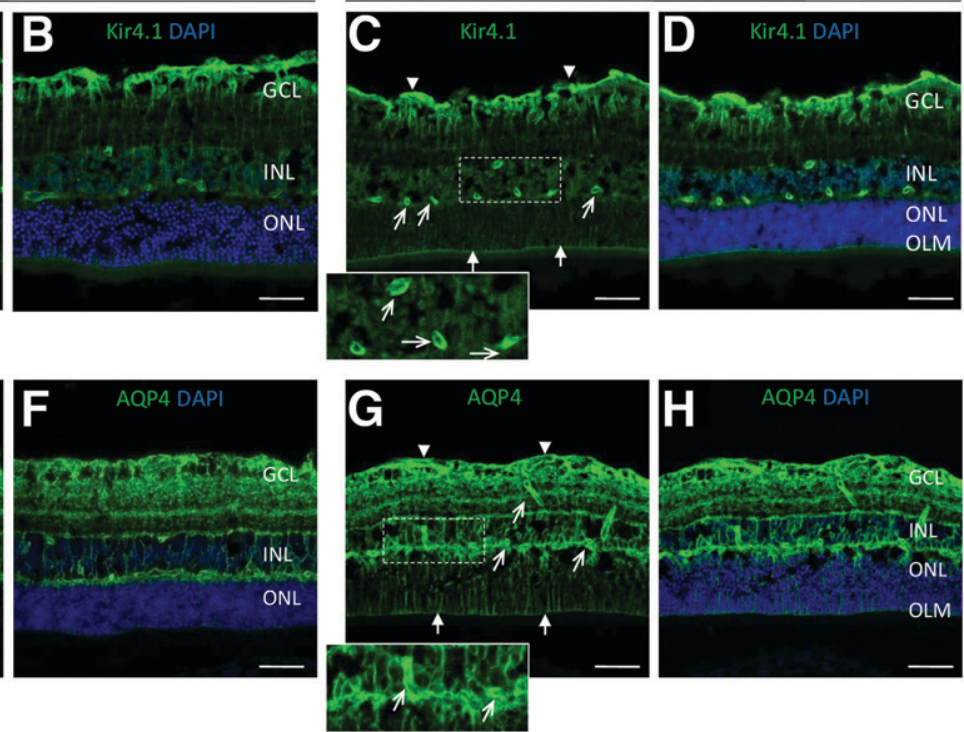
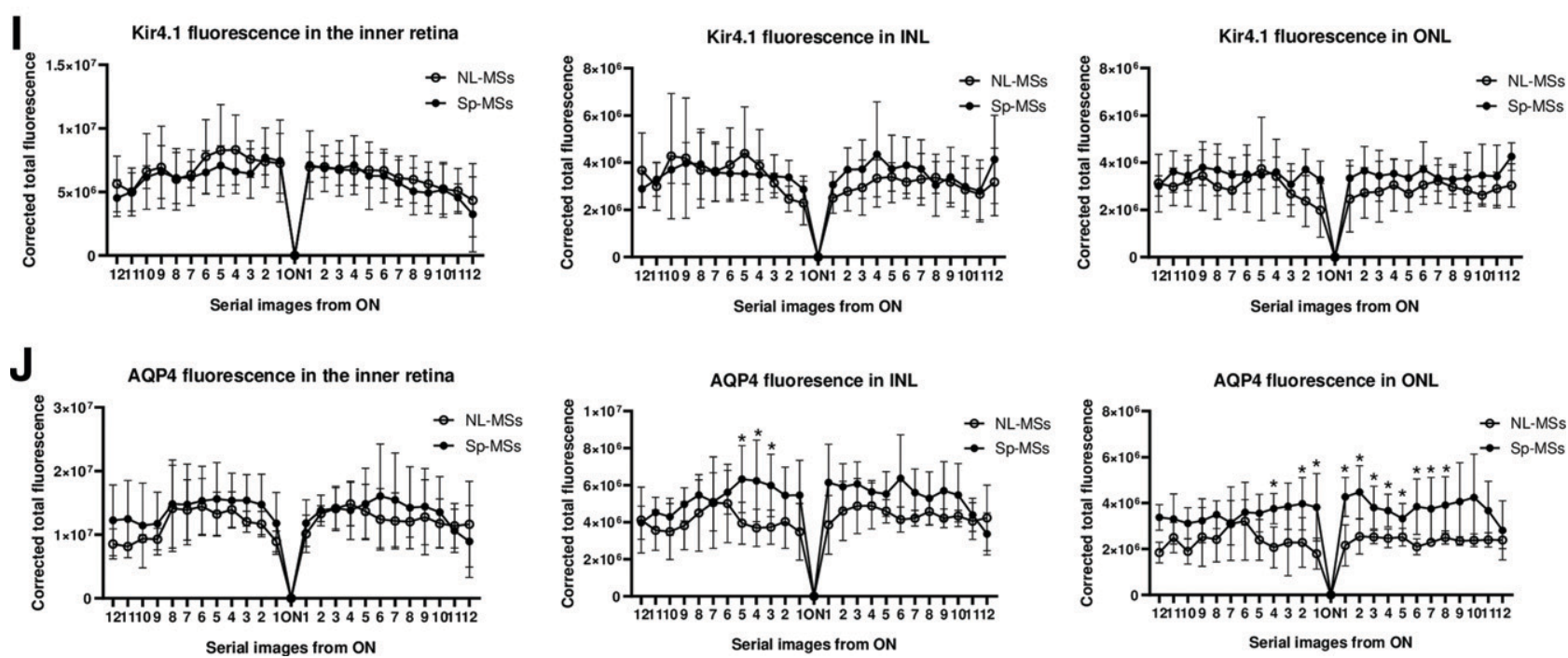

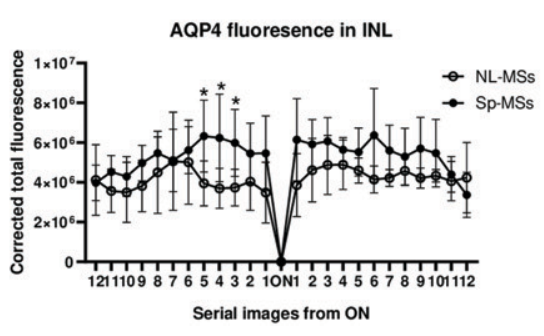

Figure 5-Sp-MSs enhance perivascular distribution of Kir4.1 and AQP4 in the retinas of old GK rats. $A-D$ : In the retinas of old (>12 months) GK rats injected with (NL-MSs), Kir4.1 (in green) localizes in the retinal Müller glial end feet (arrow heads) and faintly stains the perivascular processes ( $A$, open arrows and insert). Sp-MSs enhance the Kir4.1 expression around the vessels ( $C$, open arrows and insert) and toward outer limiting membrane (OLM) (C, closed arrows). $B$ and $D$ are merged images, with DAPI in blue. GCL, GC layer. Bar: $50 \mu$ m. $E-H$ : In the retinas of old GK rats injected with NL-MSs, AQP4 distributes in the retinal Müller glial end feet (arrow heads), and along the radial processes, the perivascular staining is weak ( $E$, open arrows and insert). In the retinas of Sp-MSs-treated GK rats, AQP4 immunostaining is generally enhanced in the Müller glial end feet ( $G$, arrow heads), around the retinal vessels ( $G$, open arrows and insert), and toward the OLM (G, closed arrows). $F$ and $H$ are merged images, with DAPI in blue. Bar: $50 \mu \mathrm{m}$. I: Quantification of Kir4.1 fluorescence shows that there is no difference in corrected total fluorescence in the inner retina (including nerve fiber layer, GCL, and IPL), INL, and ONL between NL-MSs-treated and Sp-MSs-treated groups. ON, optic nerve. $n=5$ rats per group. Data are expressed as mean \pm SD (multiple $t$ tests, corrected for multiple comparisons with the Holm-Sidak method). J: Quantification of AQP4 fluorescence shows that there is no difference in corrected total fluorescence in the inner retina between NL-MSs-treated and Sp-MSs-treated groups. But Sp-MSs increase significantly AQP4 fluorescence in the INL and ONL of posterior retinas of GK rats. $n=5$ rats per group. Data are expressed as mean $\pm \mathrm{SD} . * P<0.05$ (multiple $t$ tests, corrected for multiple comparisons using the Holm-Sidak method).

of ion and water channels distribution in macroglial cells.

\section{Local Slow Release of Spironolactone Restores the Inner and Outer Retinal Barriers}

In old GK rats, we also evaluated the effect of spironolactone on retinal barrier breakdown by measuring both albumin leakage using immunohistochemistry on retinal section and the leakage of FITC-dextran injected in vivo before sacrifice, on flat-mounted retinas. Albumin is observed preferentially around the vessels of the inner retina, in the choroid and between retinal pigment epithelium (RPE) cells in the GK diabetic rat (Fig. 7A and B). One month after intravitreous injection, Sp-MSs reduced 

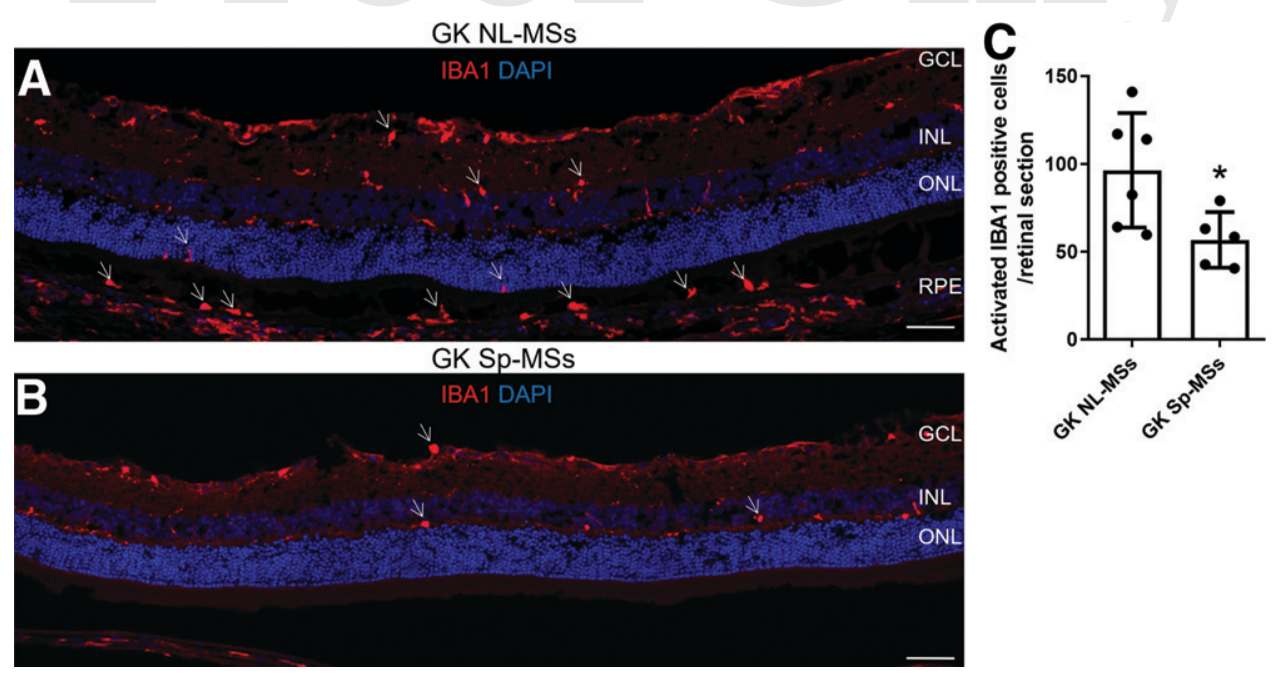

Figure 6-Sp-MSs reduce the activation of microglia/macrophages in the retina of old diabetic GK rats. A: In the retinas of old GK rats (>12 months) injected with NL-MSs, IBA1 ${ }^{+}$microglia/macrophages (in red) are activated, migrate into the outer retina up to subretinal spaces, and adhere to the RPE (arrows). B: Spironolactone released from MSs reduces the activation of microglia/macrophages and the accumulation of these cells in the subretinal spaces (arrows). GCL, GC layer. Bar: $50 \mu \mathrm{m}$. C. Quantification of activated IBA1 ${ }^{+}$cells on the retinal sections shows a significant decrease in the number of inflammatory cells in the retinas of Sp-MSs-treated old GK rats compared with NL-MSs-treated GK rats. $n=5$ rats in Sp-MSs group and 6 rats in NL-MSs group. Data are expressed as mean \pm SD. $* P<0.05$ (Mann-Whitney $U$ test).

the surface of albumin leakage on retinal sections $(P=$ 0.0152) (Fig. 7C-F) and the number of FITC-dextran leakage sites observed on retinal flat mounts $(P<0.001)$ as compared with NL-MSs (Fig. $7 G$ and $H$ ). Since macromolecule leakage drives water influx into the retina, these results suggest that the antiedematous effect of spironolactone is, at least in part, mediated by restoration of the blood-retinal barriers.

\section{Transcriptomic Signature of Spironolactone in 1-Year- Old Diabetic GK Retina}

For identification of the transcriptional regulatory mechanisms associated with the biological antiedematous and anti-inflammatory effect of spironolactone, RNA sequencing was performed on the neuroretinas of 14-month-old GK rats treated with Sp-MSs versus NL-MSs. The heat maps of genes differentially regulated by Sp-MSs as compared with NL-MSs showed a clear difference between the two conditions (Fig. 8A), with 114 genes significantly regulated between the two groups, 27 significantly downregulated, and 87 significantly upregulated (adjusted $P<0.05$ ). Pathway enrichment analysis using hallmark gene set function highlighted nine significantly regulated pathways: unfolded Q:11 protein response, ultraviolet response, G2M checkpoint, allograft rejection, interferon- $\gamma$ response, MTORC1 signaling, oxidative phosphorylation, MYC targets, and adipogenesis (Fig. $8 B$ and C). Sp-MSs regulated genes encoding proteins known to intervene in vascular permeability and retinal edema including Vldlr, Sesn2, Adcyap1, Dusp8, Pten, Slc7a1, Tjp1, Dlg1, Sema7a, and Hey1, although Vegfa was rather upregulated, suggesting an antiedematous mechanism independent of VEGF. The effect of Sp-MSs was confirmed by quantitative PCR for a number of these genes (Fig. 8D), demonstrating that spironolactone acts as a transcriptional regulator to normalize the diabetic retina.

\section{Spironolactone Preserves the Neural Retina From Excitotoxic Cell Death}

Finally, we evaluated the effect of spironolactone on excitotoxic retinal cell death using rat retinal explants submitted to NMDA, which causes cell death of the ganglion and bipolar cells within $48 \mathrm{~h}$. This model is intended to mimic the glutamate excitotoxicity, a known contributor of diabetic neurodegeneration $(38,39)$. Spironolactone protected the neural retina from necrosis measured by LDH release after 24 and $48 \mathrm{~h}(P=0.004$ and $P=0.0002$, respectively $)$ and from apoptotic cell death measured by the number of TUNEL $^{+}$cells $(P=0.045)$ (Supplementary Fig. $3 A$ and $B$ ). Spironolactone thus protects the retinal cells from excitotoxic cell death. We also quantified the total GCs on retinal histological sections of young and old rats and counted the $\mathrm{Brn} \mathrm{a}^{+}$GCs on cryosections. The number of GCs did not differ between young Wistar and GK rats, while it was sig- Q:12 nificantly reduced in old GK rats as compared with Wistar (Supplementary Fig. $3 C$ and $D$ ). We did not observe a significant increase in the number of GCs in old GK rats treated with Sp-MSs for a month (Supplementary Fig. 3D and E).

\section{Biomarkers of Diabetes Complications Regulated by MR Pathway Are Increased in Human Diabetic Retinas} The expression of two biomarkers of diabetes complications, galectin 3 (encoded by LGALS3 gene), which is associated with nephropathy and cardiovascular events $(40,41)$, and lipocalin 2 (encoded by LCN2 gene), which is associated 

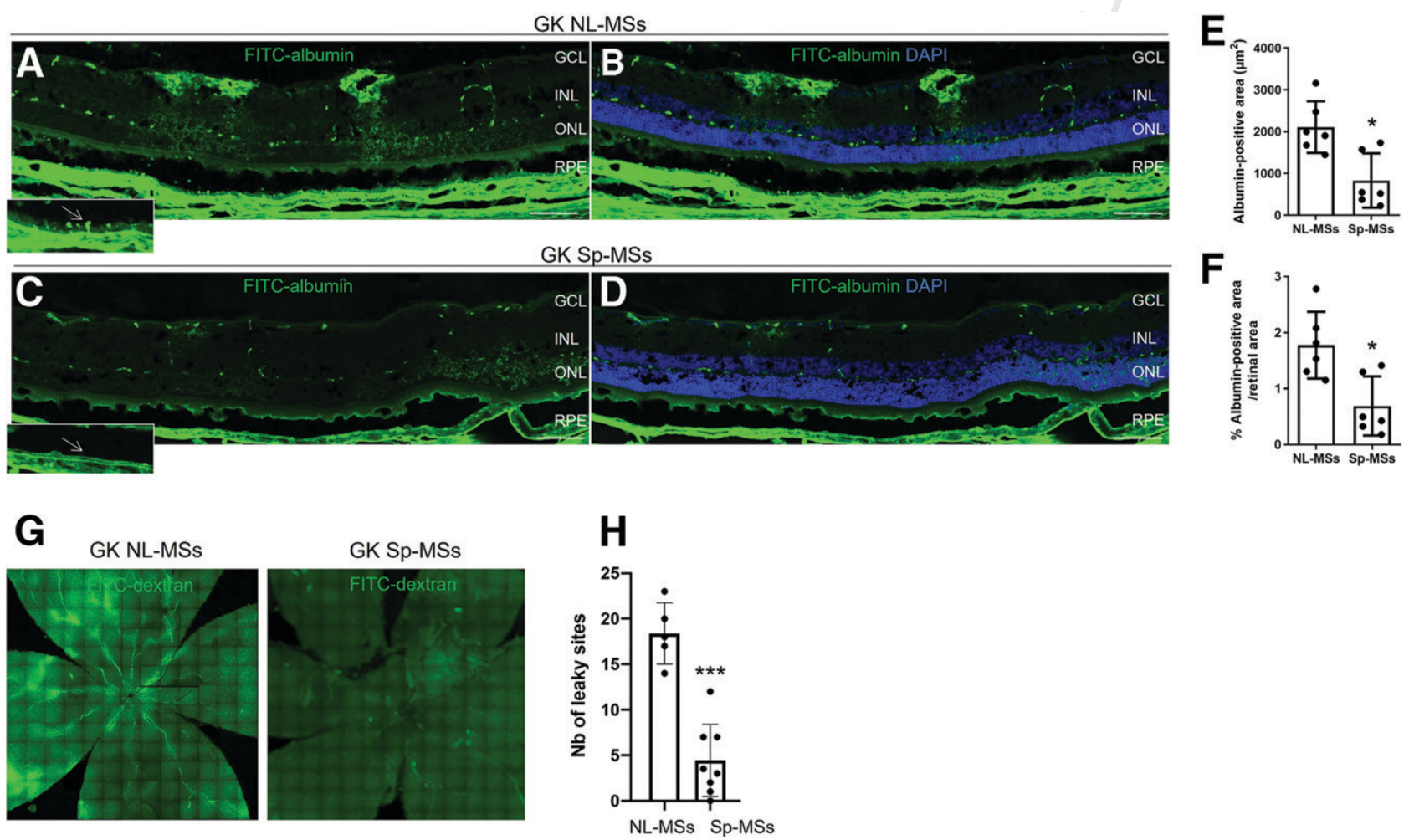

Figure 7-Sp-MSs restore the retinal-blood barrier in old GK rats. $A-D$. In the retinas of GK rats (age $>12$ months) injected with NL-MSs, immunofluorescence of FITC-albumin distributes in the retinal and choroidal vessels and in the neuroretina adjacent to vascular leakage (A). Staining of albumin can also be observed in the monolayer of RPE (arrow in insert). In the retinas of GK rats injected with Sp-MSs, extravascular albumin is decreased in the neuroretina $(C)$. No albumin is observed in the retinal pigment epithelium (arrow in insert). $B$ and $D$ are merged images, with DAPI in blue. GCL, GC layer. Bar: $100 \mu \mathrm{m}$. $E$ and F: Quantification of extravascular albumin-positive surface on retinal sections shows that Sp-MSs decrease significantly the leaking area $(E)$ and the ratio of albumin-positive surface to total analyzed retinal surface $(F) . n=6$ rats in each group. Data are expressed as mean \pm SD. ${ }^{*} P<0.05$ (Mann-Whitney $U$ test). G: Retinal flat mounts from old GK rats perfused with FITC-dextran show dextran leakage from the retinal vessels in NL-MSs-injected group (left). On the retinal flat mounts from Sp-MSs-injected GK rats, we found reduced dextran fluorescence (right). $H$ : Quantification of leaky sites on the retinal flat mounts shows that Sp-MSs treatment significantly decreases the leakage in old GK rat retinas compared with the NL-MSs control. $n=$ 5 rats in NL-MSs group and 8 rats in Sp-MSs group. Data are expressed as mean \pm SD. $* * * P<0.001$ (Mann-Whitney $U$ test). Nb, number.

with retinopathy (42), was evaluated in the retina. In the GK rats, Lcn2 and Lgals3 were highly induced in the neura retina of GK rats as compared with Wistar rats as early as 3 months of age (Supplementary Figs. $4 A$ and $5 A$ ) and Sp-MSs significantly reduced the upregulation of $L \mathrm{cn} 2$ and Lgals3 in the GK rat retinas (Supplementary Fig. $4 B$ and $5 B$ ).

Lipocalin 2 (LCN2) (the human ortholog of which is NGAL) localizing in all retinal layers and cells was enhanced in the retinas of GK rats at 6 and 12 months (Supplementary Fig. 4C). In human retinas, NGAL also distributed all over the retinal layers, including in the nuclei of cells, and seemed highly expressed in cones (Supplementary Fig. 4D). In the diabetic retinas, its expression was more intense particularly in the GCs and in the photoreceptors (Supplementary Fig. 4D).

Galectin 3 expression, restricted to the end feet of the macroglial Müller cells in the rat retinas, was moderately increased in the diabetic retinas of GK rats at 6 and 12 months (Supplementary Fig. $5 \mathrm{C}$ ). In human retinas, the glial expression of galectin 3 was highly enhanced in the diabetic retinas (Supplementary Fig. 5D) where it was also observed at the apices of the glial Muller cells in the outer retina (Supplementary Fig. 5D, insert, arrow).

NGAL/lipocalin 2 and galectin 3, two biomarkers of diabetes complications in humans, upregulated in the retina of GK rats and counterregulated by spironolactone, are present in human retina and macula and are increased in diabetic eyes, indicating that MR-downstream targets are upregulated in the retinas of humans with diabetes.

\section{DISCUSSION}

MR pathway activation is recognized in the pathogenesis of diabetes complications including coronaropathy $(10,43)$, delayed skin wound healing (44), and nephropa- Q:14 thy (14), and large clinical trials are being conducted to confirm the beneficial effect of MRA on diabetic proteinuria (16). But in diabetic retinopathy the role of the MR pathway has been poorly studied, although convergent evidence, in different animal models, shows that in the 
A

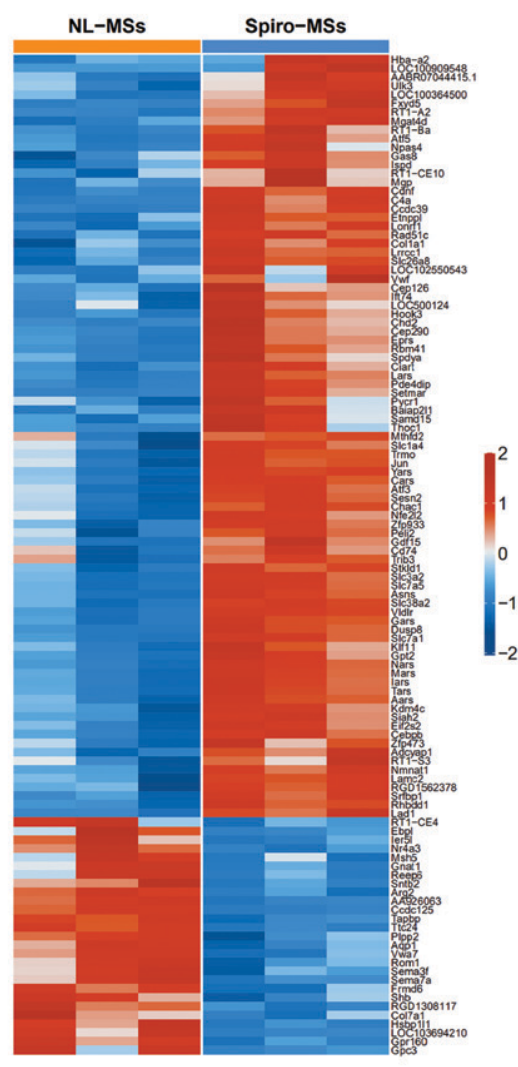

B

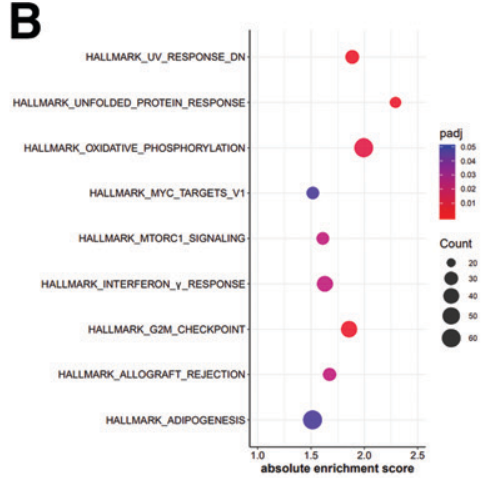

D
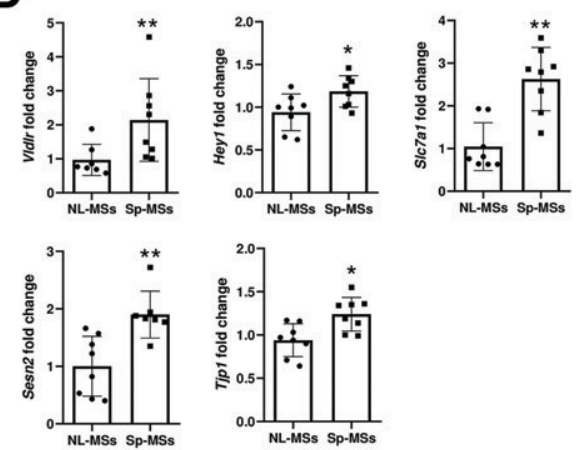
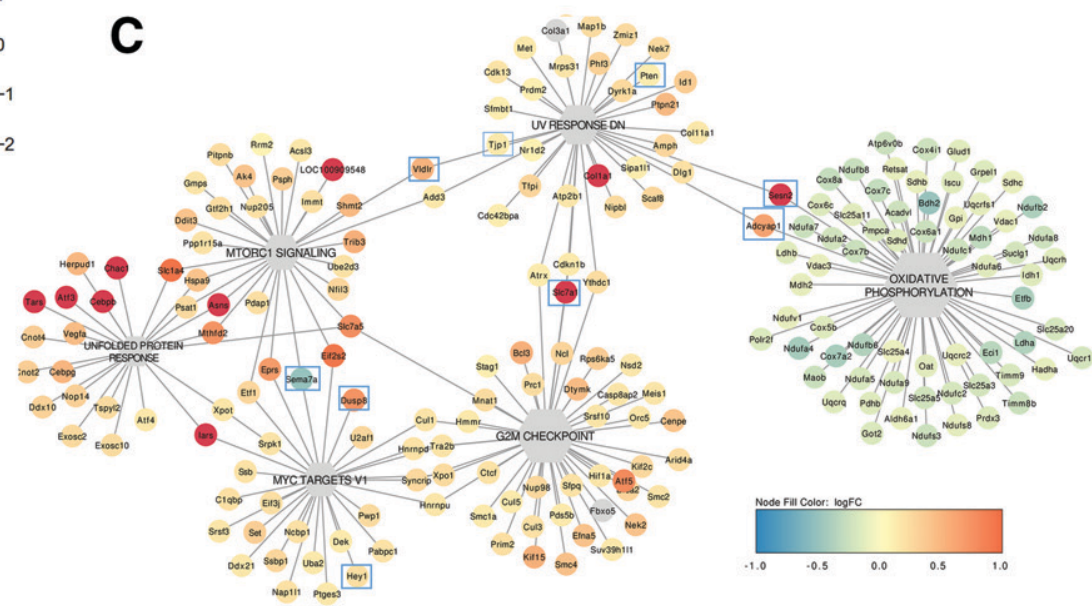

Figure 8-Transcriptomic analysis of diabetic retinas from 14-month-old GK rats injected with Sp-MSs (Spiro-MSs) vs. NL-MSs. A: The heat maps show differentially regulated genes by Sp-MSs as compared with NL-MSs. $n=3$ rats in each group. $B$ : Pathway enrichment analysis using hallmark gene set with GSEA function highlights nine significantly regulated pathways. C: Network map of the enriched

Q:27 pathways of oxidative phosphorylation, G2M checkpoint, ultraviolet response DN, MYC targets, MTORC1 signaling, and unfolded protein response. Genes involved in vascular permeability and retinal edema are highlighted in blue rectangles. D: Quantitative PCR confirms the regulation of genes VIdIr, Hey1, S/c7a1, Sesn2, and Tjp1 in the retinas of GK rats injected with Sp-MSs compared with NL-MSs. Hprt1 and $18 S$ are housekeeping genes. $n=8$ rats in each group. Data are expressed as mean \pm SD. $* P<0.05$, ${ }^{* *} P<0.01$ (Mann-Whitney $U$ test).

retina aldosterone and angiotensin II cause inflammation, oxidative stress, and pathologic angiogenesis $(18-21,29,30$, 45), which are mechanisms involved in diabetic retinopathy. In the rodent retina, MR is expressed in glial Müller cells, in interneurons and GCs, in the retinal pigment epithelium, in microglia/macrophages, and in endothelial cells of retinal and choroidal vessels $(19,21,30)$. In the human retina, we found that MR is also expressed in photoreceptors and particularly in cone photoreceptors. Previous results indicate that MR regulates locally the distribution and expression of ion and water channels in glial cells (35), also known to contribute to diabetic retinal edema. In addition, local activation of MR by aldosterone in the rat eye induced subretinal fluid accumulation, choroidal vasodilation, retinal inflammation, and oxidative stress markers $(18,19)$ and systemic slow release of aldosterone aggravated retinal edema in a rat model of vein occlusion (20). These observations show that MR overactivation is pathogenic in the retina, but which ligand could activate MR in human was unknown. Using a highly sensitive LC-MS/MS method (34), we did not detect any trace of aldosterone in the vitreous of human subjects with or without diabetes. But, interestingly, the lower cortisone-to-cortisol ratio in the vitreous of patients with diabetes, which reflects lower $11 \beta$-HSD2 activity, indicates that cortisol but not aldosterone is the ligand for MR in the diabetic retina. In the serum, low cortisone-to-cortisol ratio was previously found in patients with diabetes, and lower levels are associated with diabetes complications such as retinopathy and nephropathy (46). Thus, increased cortisol availability in the eye could activate the MR pathway in diabetes conditions.

In the GK rat retinas, $N r 3 c 2$ expression (encoding MR) is increased as compared with age-matched controls until 6 months, whereas in nondiabetic Wistar rat retinas, $N r 3 c 2$ increases only at 12 months with aging, suggesting that diabetes mimics a premature senescence (47). In human diabetic retinas, similar enhanced MR was found, particularly in the macula, where cones are concentrated. Both increased 
ligand and receptor could contribute to the overactivation of MR pathway.

The role of MR activation in the development of diabetic retinopathy has been further shown by the effect of the local delivery of the MR antagonist, spironolactone. In both young and old GK rats, the local slow release of spironolactone reversed the pathologic changes induced by diabetes. At the early stage, abnormal choroidal vasodilation was reverted, and at a later stage retinal edema was decreased. In humans, choroidal vasodilation has also been shown to precede the occurrence of microangiopathy signs (4). Although the rodent retina does not mimic completely human diabetic macular edema because rodents do not have a macula, GK rats develop retinal edema close to the human disease with change in the distribution of Kir4.1 and AQP4 and increased expression of Icam-1, Ccl2, and Plgf (35). All those changes were reverted by ocular spironolactone without glycemic control being affected, demonstrating the direct retinal effect of MRA. In addition, two markers of diabetes complications, lipocalin 2 and galectin 3, increased in the diabetic GK retina and decreased by spironolactone, were also increased in human eyes with diabetes.

There are multiple mechanisms of action of spironolactone on retinal edema (Supplementary Fig. 6). It reduces retinal inflammation at early and late stages of retinopathy through decreasing the activation and migration of macrophages/microglia and reducing the expression of inflammatory cytokines. Meanwhile, aldosterone was shown to promote an inflammatory and pro-oxidant phenotype of microglial/macrophage in a rat model of ischemic retinopathy and spironolactone reduced in vitro the ischemia-induced activation of microglial cells (48).

The restoration of retinal barriers by spironolactone shown by reduction of albumin and FITC-dextran leakage in 1-year-old GK rats was not associated with a reduction in VEGF expression. We therefore analyzed whether other molecules known to intervene in vascular permeability were regulated by spironolactone. We found that spironolactone upregulates the expression of Hey1, Vldlr, Pten, Slc7a1, Tjp1, Dlg1, and Sesn2 in the GK rat retinas (Fig. 8). Hey1, a target gene of the Notch ligand delta-like ligand 4 (Dll4), reduces vascular permeability and enhances the normal vascular maturation (49). Vldlr, encoding the VLDL receptor, is essential for maintenance of retinal permeability, as $V_{l d l r^{-1-}}$ mice show severe retinal vascular leakage (50). Pten negatively regulates the function and the phosphorylation of cavolin-1, thus reducing endothelial permeability (51). Slc7a1 encodes an interendothelial cell adhesion molecule that controls endothelial permeability (52). Tjp1, tight junction protein 1 , regulates adherens junctions (53) and discs large homolog 1 (Dlg1) controls the bloodQ:15 retinal barrier (54). Finally, the upregulation of Sesn 2 by spironolactone could reduce metabolic stress induced by hyperglycemia (55) and protect the blood-retinal barrier (56). But it is most likely that the effect of spironolactone on permeability, as is observed for other corticoids, results from a combination of mechanisms not restricted only to gene expression. Further studies are required to decipher these complex mechanisms.

On the other hand, spironolactone regulates the protein response (unfolded protein response), known to occur in the diabetic retina (57), where it plays a crucial role in the survival and activity of vascular endothelial cells, through various mechanisms including the upregulation of VEGF expression (58). Indeed, VEGF expression was not reduced by spironolactone administered in 4-month-old GK rats or in 1-year-old rats, despite the observed effect on retinal edema, suggesting an opportunity to combine spironolactone with anti-VEGF therapies.

Spironolactone effects on edema are also associated with the partial normalization of water channels along macroglial Müller cells, which play a major role in drainage mechanisms (35).

Taken together, these results indicate that MRA exerts multiple transcriptional regulations that concur with their antiedematous effects in the GK rat model. Whether similar Q:16 effects are observed in retinopathy from other experimental $\quad$ Q:17 diabetes, induced chemically or with genetic modification, particularly in type 1 diabetic models, remains to be explored.

Finally, spironolactone also protected retinal cells against excitotoxicity. Oral spironolactone did not protect in vivo against NMDA-induced cell death (59), which is expected, since spironolactone has a very poor ocular bioavailability after oral administration (28), and indicates the need for ocular delivery of MRA. In the GK rats, after 1 month of Sp-MSs exposure, we did not observe GC protection. This a:18 could be related to the late onset and short duration of Q:19 treatment, as GC loss begins early in the diabetic retina (61), or to the involvement of additional mechanisms.

In conclusion, we bring here evidence that MR pathway activation contributes to diabetic retinopathy pathogenicity in the rat and human retina and that local delivery of MRA reduces retinal edema in the GK rat through multiple mechanisms, independent from VEGF neutralization. Further studies are needed to confirm that local MRA could find a place in the therapeutic arsenal of diabetic retinopathy.

Acknowledgments. The authors acknowledge George Zadigue at the Animal Facility and Christophe Klein at the Imaging Platform of Cordeliers Research Center.

Funding. The authors thank INSERM, Agence Nationale de la Recherche (ANR Mineraloret ANR-11-BSV1-0022 and ROCK-SUR-MeR ANR-15-CE180032), the Swiss National Science Foundation (grant 156260 to C. R.), Fonda- Q:20 tion de I'Avenir, and The Abraham J. \& Phyllis Katz Foundation for financial support. This research was also supported by MAT2017- 83858-C2-1 MINECO/AEI/FEDER, Research Group UCM 920415, Innovation, Therapy and Q:21-22 Pharmaceutical Development in Ophthalmology (InnOftal), and the ISCII-FEDER RETICS (OFTARED) (RD16/0008).

Duality of Interest. No potential conflicts of interest relevant to this article were reported. 
Author Contributions. M.Z. conceived and performed experiments, supervised the students and technicians, analyzed results, and wrote the manuscript. E.G. and R.L. performed in vivo experiments and quantitative PCR. A.M. provided human eyes and did sections. F.A. provided human vitreous samples and patient information. M.B. performed the in vivo dynamic experiments. M-C.N. gave technical help for the experiments. J.G. performed the RNA-sequencing bioinformatics analysis and the corresponding graphs. K.D. performed the excitotoxic experiments. E.P. performed the LC/MS-MS steroid analysis. P.L. contributed to result analysis. I.B-0. and R.H-V. produced and characterized the MSs. F.B-C. was Principal Investigator of the study, conceived the experiments, analyzed the results, and wrote the manuscript. F.B-C. is the guarantor of this work and, as such, had full access to all the data in the study and takes responsibility for the integrity of the data and the accuracy of the data analysis.

\section{References}

1. Ogurtsova K, da Rocha Fernandes JD, Huang Y, et al. IDF Diabetes Atlas: global estimates for the prevalence of diabetes for 2015 and 2040. Diabetes Res Clin Pract 2017;128:40-50

2. Yau JWY, Rogers SL, Kawasaki R, et al.; Meta-Analysis for Eye Disease (META-EYE) Study Group. Global prevalence and major risk factors of diabetic retinopathy. Diabetes Care 2012;35:556-564

3. Bourne RRA, Jonas JB, Bron AM, et al.; Vision Loss Expert Group of the Global Burden of Disease Study. Prevalence and causes of vision loss in highincome countries and in Eastern and Central Europe in 2015: magnitude, temporal trends and projections. Br J Ophthalmol 2018;102:575-585

4. Tavares Ferreira J, Proença R, Alves M, et al. Retina and choroid of diabetic patients without observed retinal vascular changes: a longitudinal study. Am J Ophthalmol 2017;176:15-25

5. Kim K, Kim ES, Kim DG, Yu S-Y. Progressive retinal neurodegeneration and microvascular change in diabetic retinopathy: Iongitudinal study using OCT angiography. Acta Diabetol 2019;56:1275-1282

6. Lee M-W, Lee W-H, Ryu C-K, Lee Y-M, Lee Y-H, Kim J-Y. Peripapillary retinal nerve fiber layer and microvasculature in prolonged type 2 diabetes patients without clinical diabetic retinopathy. Invest Ophthalmol Vis Sci 2021;62:9

7. Cecilia $0-M$, José Alberto $\mathrm{C}-\mathrm{G}$, José $\mathrm{N}-\mathrm{P}$, et al. Oxidative stress as the main target in diabetic retinopathy pathophysiology. J Diabetes Res 2019;2019:8562408

8. Coughlin BA, Feenstra DJ, Mohr S. Müller cells and diabetic retinopathy. Vision Res 2017;139:93-100

9. Altmann C, Schmidt MHH. The role of microglia in diabetic retinopathy: inflammation, microvasculature defects and neurodegeneration. Int J Mol Sci 2018;19:E110

10. Brown SM, Meuth Al, Davis JW, Rector RS, Bender SB. Mineralocorticoid receptor antagonism reverses diabetes-related coronary vasodilator dysfunction: a unique vascular transcriptomic signature. Pharmacol Res 2018;134:100-108

11. Ferreira NS, Bruder-Nascimento $T$, Pereira CA, et al. NLRP3 inflammasome and mineralocorticoid receptors are associated with vascular dysfunction in type 2 diabetes mellitus. Cells 2019;8:1595

12. Zhang Y-Y, Li C, Yao G-F, et al. Deletion of macrophage mineralocorticoid receptor protects hepatic steatosis and insulin resistance through ER $\alpha / \mathrm{HGF} /$ Met pathway. Diabetes 2017;66:1535-1547

13. Thuzar M, Stowasser M. The mineralocorticoid receptor-an emerging player in metabolic syndrome? J Hum Hypertens 2021;35:117-123

14. Barrera-Chimal J, Girerd S, Jaisser F. Mineralocorticoid receptor antagonists and kidney diseases: pathophysiological basis. Kidney Int 2019;96:302-319

15. Sun L-J, Sun Y-N, Shan J-P, Jiang G-R. Effects of mineralocorticoid receptor antagonists on the progression of diabetic nephropathy. J Diabetes Investig 2017;8:609-618

16. Lytvyn Y, Godoy LC, Scholtes RA, van Raalte DH, Cherney DZ. Mineralocorticoid antagonism and diabetic kidney disease. Curr Diab Rep 2019; 19:4
17. Lefranc C, Friederich-Persson M, Palacios-Ramirez R, Nguyen Dinh Cat A. Mitochondrial oxidative stress in obesity: role of the mineralocorticoid receptor. J Endocrinol 2018;238:R143-R159

18. Canonica J, Mehanna C, Bonnard B, et al. Effect of acute and chronic aldosterone exposure on the retinal pigment epithelium-choroid complex in rodents. Exp Eye Res 2019;187:107747

19. Zhao M, Célérier I, Bousquet $E$, et al. Mineralocorticoid receptor is involved in rat and human ocular chorioretinopathy. J Clin Invest 2012;122:2672-2679

20. Allingham MJ, Tserentsoodol N, Saloupis P, Mettu PS, Cousins SW. Aldosterone exposure causes increased retinal edema and severe retinopathy following laser-induced retinal vein occlusion in mice. Invest Ophthalmol Vis Sci 2018;59:3355-3365

21. Wilkinson-Berka JL, Tan G, Jaworski K, Harbig J, Miller AG. Identification of a retinal aldosterone system and the protective effects of mineralocorticoid receptor antagonism on retinal vascular pathology. Circ Res 2009;104:124-133

22. Miyamoto K, Ogura $\mathrm{Y}$, Nishiwaki $\mathrm{H}$, et al. Evaluation of retinal microcirculatory alterations in the Goto-Kakizaki rat. A spontaneous model of non-insulin-dependent diabetes. Invest Ophthalmol Vis Sci 1996;37:898-905

23. Campos A, Martins J, Campos EJ, Silva R, Ambrósio AF. Choroidal and retinal structural, cellular and vascular changes in a rat model of type 2 diabetes. Biomed Pharmacother 2020;132:110811

24. Hachana S, Pouliot M, Couture R, Vaucher E. Diabetes-induced inflammation and vascular alterations in the Goto-Kakizaki rat retina. Curr Eye Res 2020;45:965-974

25. Wang W, Liu S, Qiu Z, et al. Choroidal thickness in diabetes and diabetic retinopathy: a swept source OCT study. Invest Ophthalmol Vis Sci 2020;61:29

26. Bihoreau M-T, Dumas M-E, Lathrop M, Gauguier D. Genomic regulation of type 2 diabetes endophenotypes: contribution from genetic studies in the Goto-Kakizaki rat. Biochimie 2017;143:56-65

27. Yang J, Young MJ. Mineralocorticoid receptor antagonists-pharmacodynamics and pharmacokinetic differences. Curr Opin Pharmacol 2016;27:78-85

28. Zhao M, Rodríguez-Villagra E, Kowalczuk L, et al. Tolerance of high and Iow amounts of PLGA microspheres loaded with mineralocorticoid receptor antagonist in retinal target site. J Control Release 2017;266:187-197

29. Zhao M, Mantel I, Gelize E, Li X, Xie X, Arboleda A, et al. Mineralocorticoid receptor antagonism limits experimental choroidal neovascularization and structural changes associated with neovascular age-related macular degeneration. Nat Commun 2019;10:369

30. Zhao M, Valamanesh F, Celerier I, et al. The neuroretina is a novel mineralocorticoid target: aldosterone up-regulates ion and water channels in Müller glial cells. FASEB J 2010;24:3405-3415

31. Dobin A, Davis CA, Schlesinger F, et al. STAR: ultrafast universal RNAseq aligner. Bioinformatics 2013;29:15-21

32. Li B, Dewey CN. RSEM: accurate transcript quantification from RNA-Seq data with or without a reference genome. BMC Bioinformatics 2011;12:323

33. Robinson MD, McCarthy DJ, Smyth GK. edgeR: a Bioconductor package for differential expression analysis of digital gene expression data. Bioinformatics 2010;26:139-140

34. Travers S, Martinerie L, Bouvattier C, Boileau P, Lombès M, Pussard E. Multiplexed steroid profiling of gluco- and mineralocorticoids pathways using a liquid chromatography tandem mass spectrometry method. J Steroid Biochem Mol Biol 2017;165:202-211

35. Daruich A, Matet A, Moulin A, et al. Mechanisms of macular edema: beyond the surface. Prog Retin Eye Res 2018;63:20-68

36. Hillier RJ, Ojaimi E, Wong DT, et al. Aqueous humor cytokine levels and anatomic response to intravitreal ranibizumab in diabetic macular edema. JAMA Ophthalmol 2018;136:382-388

37. Raman KS, Matsubara JA. Dysregulation of the NLRP3 Inflammasome in Q:23 Diabetic Retinopathy and Potential Therapeutic Targets. Ocul Immunol Inflamm 2020;1-9 
38. Araszkiewicz A, Zozulinska-Ziolkiewicz D. Retinal neurodegeneration in the course of diabetes-pathogenesis and clinical perspective. Curr Neuropharmacol 2016;14:805-809

39. Jindal V. Neurodegeneration as a primary change and role of neuroprotection in diabetic retinopathy. Mol Neurobiol 2015;51:878-884

40. Tan KCB, Cheung C-L, Lee ACH, Lam JKY, Wong Y, Shiu SWM. Galectin3 is independently associated with progression of nephropathy in type 2 diabetes mellitus. Diabetologia 2018;61:1212-1219

41. Tan KCB, Cheung C-L, Lee ACH, Lam JKY, Wong Y, Shiu SWM. Galectin3 and risk of cardiovascular events and all-cause mortality in type 2 diabetes. Diabetes Metab Res Rev 2019;35:e3093

42. Chung JO, Park SY, Cho DH, Chung DJ, Chung MY. Plasma neutrophil gelatinase-associated lipocalin levels are positively associated with diabetic retinopathy in patients with type 2 diabetes. Diabet Med 2016;33: 1649-1654

43. Brown NJ. Contribution of aldosterone to cardiovascular and renal inflammation and fibrosis. Nat Rev Nephrol 2013;9:459-469

44. Nguyen VT, Farman N, Palacios-Ramirez R, et al. Cutaneous wound healing in diabetic mice is improved by topical mineralocorticoid receptor blockade. J Invest Dermatol 2020;140:223-234.e7

45. Wilkinson-Berka JL, Behar-Cohen F. Angiotensin II and aldosterone: co-conspirators in ocular physiology and disease. Exp Eye Res 2020;194: 108005

46. Homma M, Tanaka A, Hino $\mathrm{K}$, et al. Assessing systemic 11betahydroxysteroid dehydrogenase with serum cortisone/cortisol ratios in healthy subjects and patients with diabetes mellitus and chronic renal failure. Metabolism 2001;50:801-804

47. Berlanga-Acosta JA, Guillén-Nieto GE, Rodríguez-Rodríguez N, et al. Cellular senescence as the pathogenic hub of diabetes-related wound chronicity. Front Endocrinol (Lausanne) 2020;11:573032

Q:24 48. Rana I, Suphapimol V, Jerome JR, Talia DM, Deliyanti D, Wilkinson-Berka JL. Angiotensin II and aldosterone activate retinal microglia. Exp Eye Res 2020;191:107902
49. Boardman R, Pang V, Malhi N, et al. Activation of Notch signaling by soluble DII4 decreases vascular permeability via a cAMP/PKA-dependent pathway. Am J Physiol Heart Circ Physiol 2019;316:H1065-H1075

50. Chen Y, Hu Y, Moiseyev G, Zhou KK, Chen D, Ma JX. Photoreceptor degeneration and retinal inflammation induced by very low-density lipoprotein receptor deficiency. Microvasc Res 2009;78:119-127

51. Gu Y, Cai R, Zhang C, et al. miR-132-3p boosts caveolae-mediated transcellular transport in glioma endothelial cells by targeting PTEN/PI3K/PKB/ Src/Cav-1 signaling pathway. FASEB J 2019;33:441-454

52. Guo L, Tian S, Chen Y, et al. CAT-1 as a novel CAM stabilizes endothelial integrity and mediates the protective actions of L-Arg via a NO-independent mechanism. J Mol Cell Cardiol 2015;87:180-191

53. Churm R, Dunseath GJ, Prior SL, Thomas RL, Banerjee S, Owens DR. Development and characterization of an in vitro system of the human retina using cultured cell lines. Clin Exp Ophthalmol 2019;47:1055-1062

54. Cho C, Wang Y, Smallwood PM, Williams J, Nathans J. Dlg1 activates beta-catenin signaling to regulate retinal angiogenesis and the blood-retina and blood-brain barriers. Elife 2019;8:e45542

55. Wang J-M, Liu B-Q, Li C, et al. Sestrin 2 protects against metabolic stress in a p53-independent manner. Biochem Biophys Res Commun 2019;513:852-856

56. Shi X, Doycheva DM, Xu L, Tang J, Yan M, Zhang JH. Sestrin2 induced by hypoxia inducible factor1 alpha protects the blood-brain barrier via inhibiting VEGF after severe hypoxic-ischemic injury in neonatal rats. Neurobiol Dis 2016;95:111-121

57. Ma JH, Wang JJ, Zhang SX. The unfolded protein response and diabetic retinopathy. J Diabetes Res 2014;2014:160140

58. Zhang SX, Ma JH, Bhatta M, Fliesler SJ, Wang JJ. The unfolded protein response in retinal vascular diseases: implications and therapeutic potential beyond protein folding. Prog Retin Eye Res 2015;45:111-131

59. Kobayashi M, Hirooka K, Ono A, Nakano Y, Nishiyama A, Tsujikawa A. The Relationship Between the Renin-Angiotensin-Aldo-sterone System and NMDA Receptor-Mediated Signal and the Pre-vention of Retinal Ganglion Cell Death. Invest Ophthalmol Vis Sci 2017;58:1397-1403
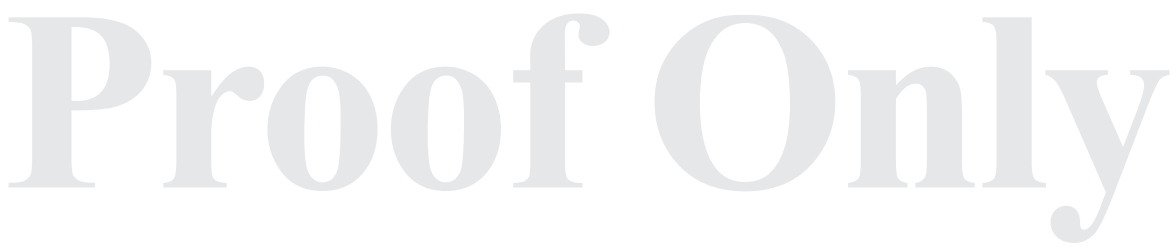


\section{AUTHOR QUERIES}

\section{AUTHOR PLEASE ANSWER ALL QUERIES}

Q1: Please verify that the information in the affiliations list is correct as edited.

Q2: Please verify that "local ethics committees (no. 2541-2015110210279792 v3, Charles Darwin University" appears as meant.

Q3: Is it correct that Vector is the name of the supplier in Les Ulis?

Q4: Does the sentence beginning "The GSEA function from the clusterProfiler package was used on a hallmark gene set ..." appear as meant?

Q5: For "M3262; SIGMA, France" is SIGMA the name of the supplier? Should this be Sigma-Aldrich or MilliporeSigma? Note that Millipore appears earlier in the paragraph, and Sigma-Aldrich Chemical Co appears later in the paragraph. Please edit as needed for consistency/accuracy of presentation.

Q6: In the sentence beginning "Ten control patients without diabetes ..." is it correct that the data in square brackets are ranges?

Q7: Please verify that "multiple $t$ tests, corrected for multiple comparisons with the Holm-Sidak method" is correct as it appears. Note that this phrasing appears several times throughout the article.

Q8: Please edit the following for clarity of expression: "associated with thickening of the outer retina witnessing retinal edema at 6 months."

Q9: Please edit the following for clarity of expression: "The expression of $I L-18$, witnessing the pathogenic activation of inflammasome..."

Q10: Does the sentence beginning "At this age, edema was more pronounced at the posterior ..." appear as meant?

Q11: Does "ultraviolet response" appear as meant for UV response? Note that this appears twice in the manuscript.

Q12: Please verify that "Wistar" is correct for WT in the sentence beginning "The number of GCs did not differ between young Wistar..."

Q13: In the sentence beginning "In the GK rats, Lcn2 and Lgals3 were highly induced . ..." is it correct that all of the figures cited are figures from the online supplementary materials?

Q14: Please verify that the following is accurate as phrased: "large clinical trials are being conducted to confirm the beneficial effect of MRA on diabetic proteinuria (16)."

Q15: Please verify that "blood-retinal barrier" appears as meant for BRB. 


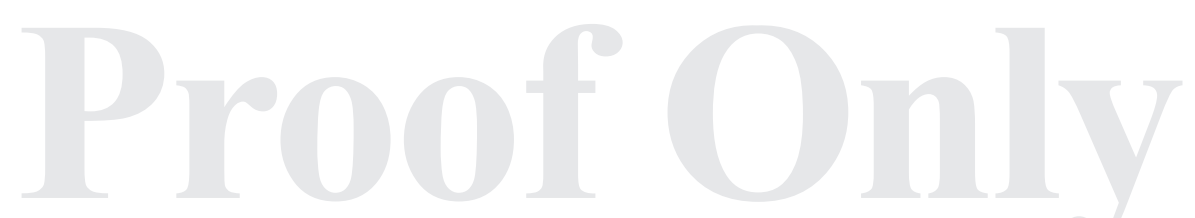

Q16: In the phrase "MRA exerts multiple transcriptional regulations that concur with their antiedematous effects," does "concur with" appear as meant?

Q17: Please verify that the sentence beginning "Whether similar effects are observed in retinopathy from other ...." is correct as it appears.

Q18: In the sentence beginning "In the GK rats, after 1 month of Sp-MSs ..." "Spi-MSs" has been edited to read "SpMSs" for consistency within the document. Please confirm that this change is correct.

Q19: The sentence beginning "This could be related to the late onset and short duration ..." contains an in-text citation for reference 61 , but there are 60 references in the reference list. Please advise.

Q20: In the Funding section, for "grant 156260 to C. R." appears, please clarify what is meant by "C. R."

Q21: Please verify that "MAT2017- 83858-C2- 1" is correct as it appears. Should there be spaces after some of the hyphens? Or should this appear "MAT2017-83858-C2-1" instead?

Q22: "MINECO/AEI/FEDER" and "ISCII-FEDER RETICS" appear in the Funding section. Please provide expansions for these for the reader, if appropriate.

Q23: Please provide a volume number for reference 37.

Q24: The manuscript contained duplicate references (numbers 35 and 48). The in-text citations and reference numbers have been renumbered accordingly. Please verify that all are correct.

Q25: In the legend to Fig. $1 B$, please verify that "GK rats ages 6 and 12 months" is correct as phrased.

Q26: Please provide a new figure for Fig. 1 so that the following is adjusted as needed: In panel C, "Henle's" is broken up so that the s appears on its own on the line below Henle'.

Q27: Please expand DN in the legend to Fig. 8, as the term appears once in the manuscript.
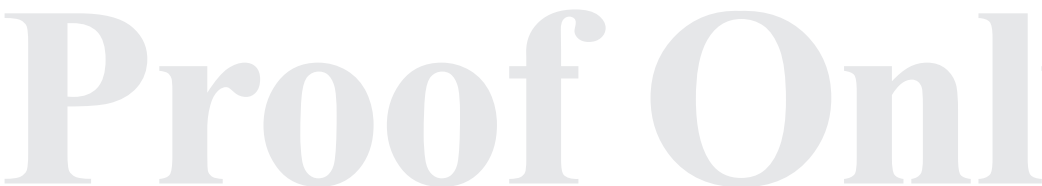